\title{
The effects of geometrical confinement and viscosity ratio on the coalescence of droplet pairs in shear flow
}

Pieter De Bruyn, Dongju Chen, Paula Moldenaers, and Ruth Cardinaels

Citation: Journal of Rheology (1978-present) 58, 1955 (2014); doi: 10.1122/1.4897266

View online: http://dx.doi.org/10.1122/1.4897266

View Table of Contents: http://scitation.aip.org/content/sor/journal/jor2/58/6?ver=pdfcov

Published by the The Society of Rheology

\section{Articles you may be interested in}

Droplet dynamics in mixed flow conditions: Effect of shear/elongation balance and viscosity ratio

J. Rheol. 54, 1285 (2010); 10.1122/1.3490661

Effects of viscosity ratio and three dimensional positioning on hydrodynamic interactions between two viscous drops in a shear flow at finite inertia

Phys. Fluids 21, 103303 (2009); 10.1063/1.3253351

Oscillatory shear induced droplet deformation and breakup in immiscible polymer blends Phys. Fluids 21, 063102 (2009); 10.1063/1.3153304

Effect of confinement and viscosity ratio on the dynamics of single droplets during transient shear flow

J. Rheol. 52, 1459 (2008); 10.1122/1.2978956

Drop formation and breakup of low viscosity elastic fluids: Effects of molecular weight and concentration

Phys. Fluids 18, 043101 (2006); 10.1063/1.2190469

\section{囯 Re-register for Table of Content Alerts}




\title{
The effects of geometrical confinement and viscosity ratio on the coalescence of droplet pairs in shear flow
}

\author{
Pieter De Bruyn, Dongju Chen, Paula Moldenaers, ${ }^{\text {a) }}$ and Ruth Cardinaels \\ Department of Chemical Engineering, KU Leuven, Willem de Croylaan 46, \\ B-3001 Leuven, Belgium
}

(Received 9 April 2014; final revision received 20 September 2014;

published 17 October 2014)

\begin{abstract}
Synopsis
The effects of geometrical confinement and viscosity ratio on droplet coalescence in shear flow are experimentally investigated by means of a counter rotating parallel plate device, equipped with a microscope. The ratio of droplet diameter to gap spacing is varied between 0.03 and 0.33 to study both bulk and confined conditions. Three grades of a Newtonian droplet material are combined with a Newtonian matrix, resulting in three different viscosity ratios, namely, $0.1,1.1$, and 2.6. The effects of confinement are qualitatively similar for all three viscosity ratios. For each system, confinement decreases the coalescence angle and renders coalescence possible up to higher capillary numbers and initial offsets. Moreover, for all three viscosity ratios, confinement induces a lower initial offset boundary below which the approaching droplets reverse flow direction without coalescence. However, there are quantitative differences between the systems. With increasing viscosity ratio, the critical capillary number and critical upper and lower offset boundaries decrease. Since the decrease of the upper offset boundary is more predominant, the coalescence efficiency decreases with viscosity ratio. The droplet trajectories of interacting droplets are affected by both the viscosity ratio and geometrical confinement, which clearly has implications on the coalescence behavior. (C) 2014 The Society of Rheology.

[http://dx.doi.org/10.1122/1.4897266]
\end{abstract}

\section{INTRODUCTION}

Polymer blending is a fast and economic route to produce new materials with enhanced properties. The properties of an immiscible blend not only depend on the properties of the component materials but are also affected by the droplet size and its distribution. The latter is determined by a balance between droplet breakup and coalescence. These processes can occur simultaneously when the blends are subjected to complex flow fields during processing. Nowadays, there is a growing interest in small scale mixing equipment [Son (2009); Cassagnau and Fenouillot (2004)]. Especially during the research and development stage of polymer blends and composites, these devices can be very useful. Due to the small scale of these processing devices, interactions of the droplets with the walls cannot be excluded. Although the flow in mixing devices is in principle a

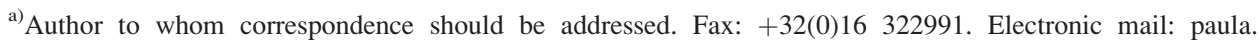
moldenaers@cit.kuleuven.be
} 
combination of shear and extension, shear flow is often the main flow component. Hence, to gain fundamental insight in the effects of wall proximity (=confinement effects) during processing, a large amount of research has been devoted to morphology development in confined shear flow.

For droplet-matrix systems in confined shear flow, interesting new phenomena were observed. For example, when shearing concentrated blends in a confined parallel plate geometry, Migler (2001) found a droplet-to-string transition with a pearl necklace morphology as an intermediate regime. Pathak et al. (2002) reported that pearl necklaces can occur as a stable morphology; layered morphologies can also form as a result of droplet interactions. Moreover, for low viscosity ratio systems shear-induced banding, i.e., the formation of alternating regions of high and low volume fractions of droplets, was reported due to confinement [Caserta et al. (2008); Caserta and Guido (2012)]. These morphologies certainly have potential for applications such as the production of wires and fibers [Migler (2001)]. However, fundamental insight in the formation of these superstructures is still lacking. Obviously, coalescence plays a key role in their formation. To develop a thorough understanding of morphology development in confined flow, studies in which either droplet breakup or droplet coalescence are studied separately, are essential.

For a fundamental investigation of the effects of confinement on droplet coalescence, the study of the interaction and coalescence of isolated droplet pairs is a good approach. Coalescence of an isolated droplet pair has typically been described as a three stage process [Chesters (1991)]. The first stage is the approach of the droplets as a result of the flow. When the distance between the droplets becomes small, the matrix fluid in between the droplets needs to be squeezed out. This is the beginning of the second stage: the film drainage. When the distance between the two droplet surfaces becomes sufficiently small, van der Waals forces can destabilize the film, leading to film rupture, which is the third and final stage. This final film rupture stage occurs almost instantaneously. The interaction time of droplets in a glancing collision will only be sufficient to allow for film drainage up to the rupture stage, and thus coalescence, if the shear rate is sufficiently small [see, e.g., Yang et al. (2001)]. Similar to the deformation and breakup of single droplets, as pioneered by Taylor (1932), the effect of shear rate on droplet coalescence can be described by means of the capillary number $C a\left(=\eta_{m} \cdot \dot{\gamma} \cdot R / \Gamma\right.$, with $\eta_{m}$ being the matrix viscosity, $\dot{\gamma}$ being the shear rate, $R$ being the droplet radius, and $\Gamma$ being the interfacial tension). As a consequence, there is a critical capillary number below which coalescence occurs.

The effect of viscosity ratio on droplet coalescence has already been experimentally investigated by Yoon et al. (2005) for pure extensional flow. They found that the drainage time increases and the critical capillary number decreases with increasing viscosity ratio. These observations were later confirmed by means of numerical simulations [Yoon et al. (2007)]. Moreover, the effect of initial offset between the droplets in the velocity gradient direction was investigated. For a viscosity ratio of 0.1 , the critical capillary number decreases monotonically with increasing offset [Yang et al. (2001)]. On the other hand, when the viscosity ratio is larger than 0.1 , the critical capillary number only decreases with increasing offset for the smallest offsets and then increases with increasing offset until a critical offset is reached above which no coalescence is possible [Leal (2004); Yoon et al. (2005)]. This phenomenon is caused by the fact that for the largest offsets there is a shift of the coalescence angle to the extensional quadrant, so coalescence occurs while the droplets are being pulled apart by the external flow. The experimental studies of Tretheway et al. (1999) showed that the trajectories of interacting droplets in extensional flow depend on the viscosity ratio. Mousa et al. (2002) calculated 
the coalescence efficiencies for different viscosity ratios in shear flow using the partially mobile interface model and found a decrease of the coalescence efficiency with viscosity ratio. Minale et al. (1997) experimentally investigated the effect of viscosity ratio on the average droplet size in concentrated sheared blends and found that the accuracy of the fully mobile drainage model increases with decreasing viscosity ratio, whereas the opposite is true for the immobile drainage model. Also Lyu et al. (2000) and Caserta et al. (2006) investigated the effect of viscosity ratio in concentrated sheared blends and found that the coalescence rate slowed down with increasing viscosity ratio. Loewenberg and Hinch (1997) numerically investigated the collision of two deformable drops in shear flow at different viscosity ratios. More recently, Singh and Sarkar (2009) and Bayareh and Mortazavi (2011) numerically studied the effect of viscosity ratio on droplet collisions at finite inertia. Singh and Sarkar (2009) found reversing droplet trajectories in the presence of inertia. Moreover, it was found that the tendency of the droplets to reverse decreases with increasing viscosity ratio.

The first experimental study dealing with the effects of confinement on the coalescence of sheared droplets was performed by Chen et al. (2009). They systematically investigated the effects of geometrical confinement on the coalescence angles and critical $\mathrm{Ca}$ numbers in pure shear flow for two equal-sized droplets with a droplet-to-matrix viscosity ratio of one. It was found that a low degree of confinement only decreases the orientation angle at which the droplets coalesce, whereas the critical conditions for coalescence remain the same. On the other hand, when the confinement ratio $(=2 R / H$ with $R$ being the droplet radius and $H$ being the gap width) is larger than 0.2 , the critical capillary number up to which coalescence can occur, increases. Thus, confinement can clearly promote droplet coalescence. De Bruyn et al. (2013) further explored the effects of geometrical confinement on the coalescence of droplet pairs by varying the initial offset between the droplet centers in the velocity gradient direction. For droplet pairs with a droplet-to-matrix viscosity ratio of 0.1 , a new phenomenon of reversing trajectories for droplets with low initial offsets was demonstrated. Since the droplets reverse flow direction without coalescence, the reversal induces a lower initial offset boundary for coalescence in confinement. In addition, it was observed that due to confinement coalescence is possible up to higher initial offsets. Reversing trajectories were also encountered in simulations of solid particles interacting in confined shear flow [Zurita-Gotor et al. (2007)]. Recently, Sarkar and Singh (2013) numerically investigated the effect of confinement on droplet collisions in shear flow. They studied collisions that do not result in coalescence and focused on the wall-induced migration of the two droplets to the center of the gap after collision. Shardt et al. (2013) performed a limited number of numerical simulations of droplet coalescence in confinement. They observed that above a certain confinement ratio, the critical capillary number increases with confinement ratio, which is in agreement with the data of Chen et al. (2009).

The combined effects of confinement and viscosity ratio have already been investigated for droplet breakup. It has been shown that confinement effects on droplet breakup can be qualitatively different, depending on the viscosity ratio [Vananroye et al. (2006)]. For viscosity ratios below one, confinement suppresses breakup, whereas for viscosity ratios above one, breakup is promoted by confinement [Vananroye et al. (2006)]. Furthermore, Cardinaels and Moldenaers (2011) studied the combined effect of confinement and viscoelasticity of one of the components. They found that droplet breakup in confined shear flow is also strongly dependent on the viscoelasticity of the components. More specifically, in confinement matrix viscoelasticity causes breakup at much lower critical $\mathrm{Ca}$ numbers as compared to that for droplets in a Newtonian matrix, whereas the viscoelastic effects in bulk conditions were limited [Cardinaels and Moldenaers (2011)]. 
These studies clearly show that confinement effects can substantially differ, depending on the component rheology.

Although it has been demonstrated in previous studies that geometrical confinement can promote droplet coalescence, it is not clear up to now, how the coalescence of confined droplets is affected by viscosity ratio. In the present study, the effects of geometrical confinement on droplet coalescence in shear flow are investigated for three different viscosity ratios $\lambda$, namely, $\lambda$ around $1, \lambda$ larger than 1 , and $\lambda$ smaller than one. A complete study of the effects of confinement on the coalescence process including critical $\mathrm{Ca}$ numbers, critical offsets, coalescence angles, droplet trajectories, and doublet rotation speeds is presented for the first time. Finally, 2D numerical simulations, providing streamlines and stresses on the droplet interfaces in bulk and confined conditions, allow to improve the present understanding of the hydrodynamics of the confinement and viscosity ratio effects.

\section{MATERIALS AND METHODS}

\section{A. Materials}

Polyisobutylene (PIB Glissopal V-190 from BASF) with a viscosity $\eta_{m}$ of $10.5 \mathrm{~Pa} \cdot \mathrm{s}$ at $27^{\circ} \mathrm{C}$ was used as the matrix phase. To provide systems with different viscosity ratios, three different grades of polydimethylsiloxane (PDMS Rhodorsil 47 V1000 and V12500 from Rhodia and PDMS Silbione 70047 V30000 from Bluestar Silicones) were used as the droplet phase. Table I summarizes the viscosities $\eta_{d}$ of the three PDMS grades at $27^{\circ} \mathrm{C}$ and the resulting viscosity ratios $\lambda$ of the corresponding droplet-matrix pairs. In the relevant experimental shear rate range, all materials behave Newtonian and there is no measurable elasticity. The interfacial tension $\Gamma$ between PDMS and PIB was obtained by fitting deformation data of a single droplet at low capillary numbers with the small deformation theory of Greco [Guido et al. (2003)]. This resulted in a value of $1.8 \mathrm{mN} / \mathrm{m}$, irrespective of the PDMS grade. PIB is known to be slightly soluble in PDMS, whereas PDMS is totally insoluble in PIB [Guido et al. (1999)]. Consequently, systems consisting of PDMS droplets in a PIB matrix can be considered completely immiscible. Due to the small density difference between PDMS and PIB $\left(0.08 \mathrm{~g} \cdot \mathrm{cm}^{-3}\right)$ [Guido and Simeone (1998)], buoyancy effects are expected to be negligible.

\section{B. Experimental setup}

The experiments were performed with a counter rotating parallel plate shear flow device (based on an Anton Paar MCR 300 rheometer). This device consists of two parallel quartz plates with a diameter of $50 \mathrm{~mm}$, which can rotate in opposite direction. Hence, in between the plates a stagnation plane with a velocity of zero is created, in which interacting droplets can be easily visualized with a nonmoving microscope setup. A detailed description of the device can be found elsewhere [Vananroye et al. (2006); Verhulst et al. (2007)]. The optical train uses a combination of a stereo microscope (Wild M5A)

TABLE I. Zero-shear viscosities of the different PDMS grades at $27^{\circ} \mathrm{C}$ and the resulting viscosity ratios of the blends at $27^{\circ} \mathrm{C}$.

\begin{tabular}{lcc}
\hline \hline PDMS grade & $\eta_{d}\left(27^{\circ} \mathrm{C}\right)(\mathrm{Pa} \mathrm{s})$ & $\lambda=\eta_{d} / \eta_{m}\left(27^{\circ} \mathrm{C}\right)$ \\
\hline PDMS Rhodorsil 47 V1000 & 0.95 & 0.1 \\
PDMS Rhodorsil 47 V12500 & 11.2 & 1.1 \\
PDMS Silbione 70047 V30000 & 27.8 & 2.6 \\
\hline \hline
\end{tabular}


and a digital camera (Basler A631fc) for visualization, resulting in a resolution of 1.34 pixels $/ \mu \mathrm{m}$. The droplet pair was visualized in the velocity-velocity gradient plane and images were taken with Streampix Digital Video Recording Software (Norpix).

\section{Terminology}

A schematic of two colliding droplets in shear flow is shown in Fig. 1. The $x$-axis is the velocity direction, the $y$-axis is the velocity gradient direction. The relative trajectory of two colliding droplets is expressed by the distance between the droplet centers in the flow direction $\Delta X=\left(X_{2}-X_{1}\right)$ and in the velocity gradient direction $\Delta Y=\left(Y_{2}-Y_{1}\right)$, with $\left(X_{1}, Y_{1}\right)$ and $\left(X_{2}, Y_{2}\right)$ the coordinates of the droplet centers. The center-to-center distance between the droplets is $d$. The orientation angle $\theta$ is the angle between the flow direction and the line connecting the droplet centers. For all experiments, the two droplets are always placed approximately symmetric with respect to the gap center.

\section{Experimental protocol}

A homemade injection device was used to inject a PDMS droplet of the required size in the PIB matrix. Subsequently, this droplet was broken up in two daughter droplets by applying a strong shear flow. This method results in two droplets with a difference in diameter of less than a few percent. To obtain the desired initial offset $\Delta Y$ between the droplets, the following protocol was employed: A shear rate is applied which results in an approach of the droplets and subsequent rotation of the droplets over each other. When during the rotation the desired offset is reached, the flow is stopped and the flow direction is reversed. The flow is stopped again when the desired distance $\Delta X$ is reached. The initial dimensionless distance in the velocity direction $\Delta X_{\text {in }} / 2 R$ was fixed here at 1.6 for all experiments. After retraction of the droplets to their spherical shape, droplet collision was induced by applying a low shear rate, leading to creeping flow conditions. This methodology is similar to that used by Guido and Simeone (1998). In this work, a gap spacing $H$ of $3 \mathrm{~mm}$ is used to represent bulk conditions and a gap spacing $H$ of either 0.5 or $1 \mathrm{~mm}$ is used to represent confined conditions. The droplets are non-Brownian with diameters between 85 and $315 \mu \mathrm{m}$. This leads to confinement ratios $2 R / H$ up to 0.33 .

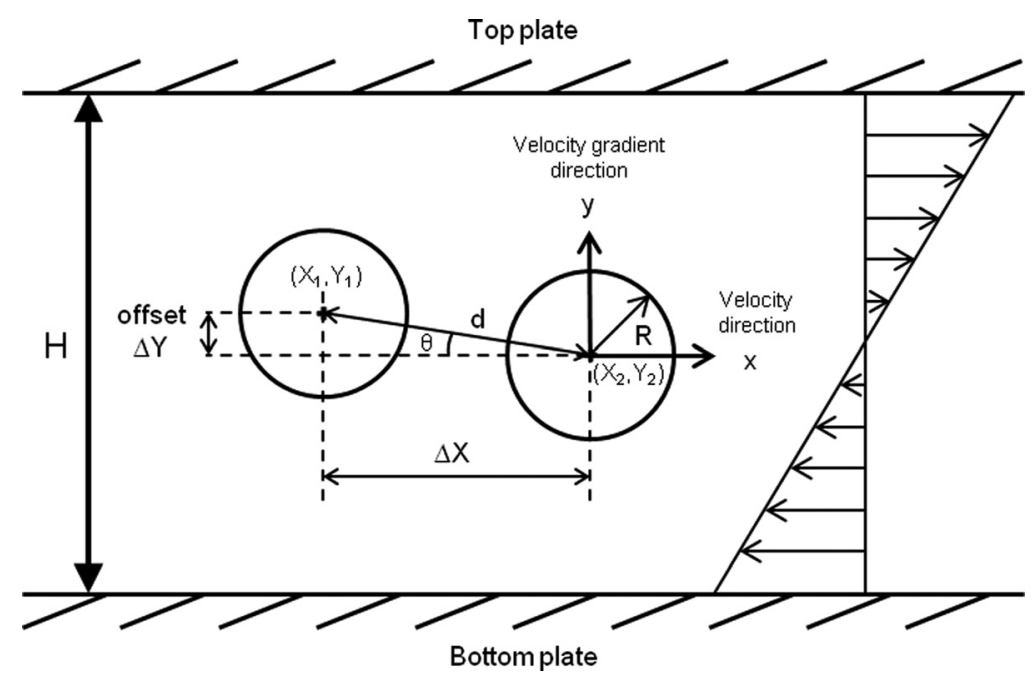

FIG. 1. Schematical representation of two colliding droplets in shear flow. 
During all experiments, the sample temperature was kept constant at $27^{\circ} \mathrm{C}$ by controlling the room temperature.

\section{E. Data analysis}

For the image analysis, ImageJ Software was used. As a consequence of the curvature of the cup around the bottom plate [see schematic of device in Vananroye et al. (2006)], the images in the velocity-velocity gradient plane are slightly elongated in the velocity direction. Hence, the first step of the image analysis is a rescaling of the images in the velocity direction. For the next step, the detection of the droplet edges, a Laplace operator was used. For images in which the two droplets are in apparent contact, a watershed algorithm was applied to separate the images of both droplets from each other. This algorithm generates a white line between the cusps on both sides of the doublet. In a final step, an ellipse was fitted on the droplet contour.

\section{F. Numerical simulations in 2D}

In addition to the experiments, numerical simulations of two interacting droplets in shear flow were performed in 2D. The simulation results are presented in Figs. 12 and 15. The computational domain is a rectangle with a length of at least 30 times the droplet radius. It was verified that, under these conditions, simulation results are not affected by the length of the computational domain. As in the experiments, the gap height $H$ is varied between 0.5 and $3 \mathrm{~mm}$. The upper wall of the channel is moving with a velocity $U$, and the lower wall moves with the same velocity but in the opposite direction, resulting in a shear rate $\dot{\gamma}=2 U / H$. As boundary condition at the inlet and outlet a linear velocity profile, characteristic for shear flow between two parallel plates, was imposed. The two droplets are placed symmetric with respect to the gap center. Before the start of the simulation, the two droplets are spherical and the startup of the flow is instantaneous. Both the matrix and droplet fluid are incompressible and density-matched. At the droplet interfaces continuity of velocity and a balance between interfacial tension and normal stresses is assumed,

$$
\begin{gathered}
\boldsymbol{u}_{m}=\boldsymbol{u}_{d} \\
\boldsymbol{n} \cdot\left(\boldsymbol{T}_{m}-\boldsymbol{T}_{d}\right)=\Gamma(\nabla \cdot \boldsymbol{n}) \boldsymbol{n},
\end{gathered}
$$

where $\boldsymbol{n}$ is the normal vector at the interface and $\boldsymbol{T}$ is the total stress in each fluid,

$$
\boldsymbol{T}=-p \boldsymbol{I}+\eta\left(\nabla \boldsymbol{u}+(\nabla \boldsymbol{u})^{T}\right) .
$$

The values for the viscosities and the interfacial tension were taken equal to the experimental values. The equations that have to be solved in both fluid domains are the continuity equation and the momentum balance,

$$
\begin{gathered}
\nabla \cdot \boldsymbol{u}=0 \\
\rho\left(\frac{\partial \boldsymbol{u}}{\partial \tau}+\boldsymbol{u} \cdot \nabla \boldsymbol{u}\right)=\nabla \cdot\left(-p \boldsymbol{I}+\eta\left(\nabla \boldsymbol{u}+(\nabla \boldsymbol{u})^{T}\right)\right) .
\end{gathered}
$$

To discretize these equations, a finite element method from the COMSOL Multiphysics 4.2 package was used. The matrix and droplet domains were discretized by means of a 
fine mesh with element sizes between 5 and $17.5 \mu \mathrm{m}$ for the simulations with a gap of $0.5 \mathrm{~mm}$ and between 30 and $105 \mu \mathrm{m}$ for the simulations with a gap of $3 \mathrm{~mm}$. In both cases, this resulted in about 30 layers of triangular mesh elements across the gap width. At the moving walls, an additional boundary layer consisting of six layers of quadrilateral mesh elements was inserted. Along the droplet interfaces, an extra fine triangular mesh with 160 vertices per droplet interface was used. This resulted in a finer mesh in between and around the droplets, which gradually coarsened to the mesh size of the matrix and droplet domains. The degree of refinement was chosen such that, either a further refinement of the overall size of the mesh elements, or an increase of the number of mesh elements along the droplet interface, does not have a noticeable effect on the presented simulation results (Figs. 12 and 15). In these simulations, a moving mesh method using the arbitrary Langrangian-Eulerian formulation is used, which allows the droplets to deform. However, because in this method the mesh is fixed to the interface, topological changes such as droplet coalescence cannot be simulated. It should be stressed that, given the intrinsic nonaxisymmetrix 3D nature of the problem [Loewenberg and Hinch (1997)], a 2D simulation is only a rough approximation of a real droplet collision. For example, the 2D simulations show deviations of $9 \%$ and $48 \%$ with respect to 3D simulation results of Aggarwal and Sarkar (2007) for, respectively, the droplet deformation and peak value of the normal stress on the droplet interface of a single droplet with $\lambda=1$ deformed in shear flow at $C a=0.2$. However, the stress profile along the droplet interface qualitatively corresponds to that obtained from the $3 \mathrm{D}$ simulations. Although the simulations allow to shed light on the origins of the confinement effects, the absolute values of the simulated stresses may thus not quantitatively match those in the real 3D system.

\section{RESULTS AND DISCUSSION}

\section{A. Critical capillary number for coalescence}

For droplet-matrix systems with a viscosity ratio of 1.1, it was shown that geometrical confinement can substantially increase the critical $C a$ number and hence, promote coalescence [Chen et al. (2009)]. In order to assess the effect of viscosity ratio on these phenomena, critical $\mathrm{Ca}$ numbers as a function of droplet diameter were determined for systems with viscosity ratios $\lambda$ of 0.1 and 2.6 and compared with the values for a viscosity ratio of 1.1 from Chen et al. (2009). Figure 2 displays the results both for unconfined and confined droplets. To determine the critical $\mathrm{Ca}$ number for a certain droplet size, droplet collisions were performed for a range of Ca numbers. First a collision experiment was performed at a small $C a$ number for which coalescence is expected. Consequently the experiment is repeated with a new droplet pair, but each time the $C a$ number is increased with steps of maximum 0.001, until at a certain $C a$ number the droplets rotate over each other without coalescence. The symbols in Fig. 2 represent the highest $\mathrm{Ca}$ number for which coalescence still occurred. The initial dimensionless offset $\Delta Y_{\text {in }} / 2 R$ is fixed at 0.23 for $\lambda=0.1$ and 2.6. For the measurements at viscosity ratio $\lambda=1.1$, the initial dimensionless offset was 0.16 . The filled symbols are the critical $\mathrm{Ca}$ numbers at a gap of $3 \mathrm{~mm}$ (or $4 \mathrm{~mm}$ for the largest droplet sizes), which results in confinement ratios $2 R / H$ below 0.08 . The droplet behavior at this small confinement ratio can be considered as the reference case of unconfined shear flow. The open symbols are the critical $C a$ numbers at a gap of $1 \mathrm{~mm}$, resulting in confinement ratios $2 R / H$ between 0.08 and 0.32 . This dataset represents more confined conditions. In the unconfined case the critical capillary number decreases with droplet size for all three viscosity ratios. The fact that the critical capillary number decreases with droplet size is in qualitative agreement with literature 


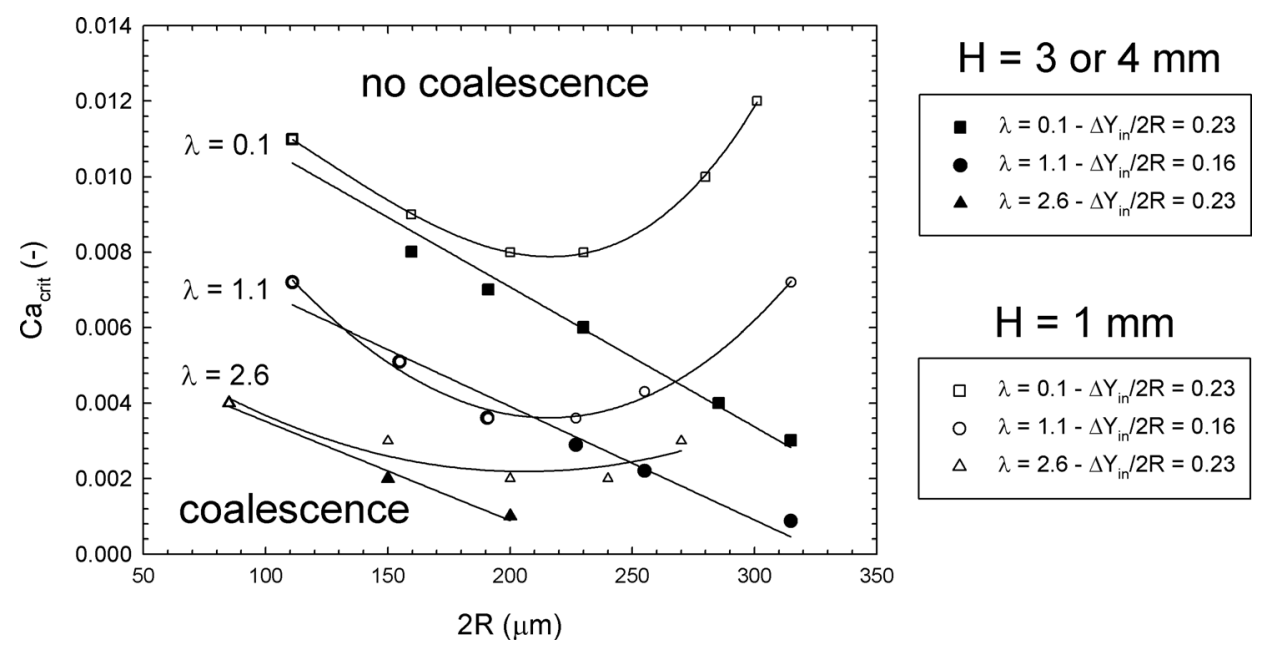

FIG. 2. Critical capillary number for coalescence $C a_{\text {crit }}$ as a function of droplet diameter $2 R$. Filled symbols: $H=3$ or $4 \mathrm{~mm}$. Open symbols: $H=1 \mathrm{~mm}$. For $\lambda=0.1$ and 2.6 the initial dimensionless offset $\Delta Y_{\mathrm{in}} / 2 R=0.23$, for $\lambda=1.1$ the initial dimensionless offset $\Delta Y_{\text {in }} / 2 R=0.16$. The symbols represent the highest $C a$ for which coalescence occurred. These values were determined by varying the $C a$ number in steps of maximum 0.001 . Lines to guide the eye.

results for mixed linear flows [Hu et al. (2000); Park et al. (2003); Leal (2004)]. It is also clear from Fig. 2 that in unconfined conditions the critical $C a$ number decreases with increasing viscosity ratio. Such a decrease of the critical $C a$ number with increasing viscosity ratio has already been reported in literature for droplet coalescence in extensional flow [Hu et al. (2000); Yoon et al. (2005)]. It should be noted here that due to the decrease of the critical $C a$ number with viscosity ratio, coalescence does not occur within the experimentally accessible range of $\mathrm{Ca}$ numbers for systems with viscosity ratios higher than those shown in Fig. 2.

In confined conditions, the trend is clearly different. Up to a droplet diameter $2 R$ of approximately $200 \mu \mathrm{m}$ and thus a confinement ratio of $2 R / H=0.2$, the critical capillary number is almost the same as for unconfined conditions. On the other hand, for droplets with a diameter $2 R$ larger than $200 \mu \mathrm{m}$ and thus a confinement ratio larger than $2 R / H=0.2$, the critical capillary number increases with droplet size. As a result, for these big droplets there is a range of capillary numbers for which coalescence is not possible in bulk conditions, but becomes possible in confined conditions. Clearly in this case confinement promotes coalescence. Moreover, the magnitude of the confinement effect on the critical $\mathrm{Ca}$ number for coalescence is almost equal for the three viscosity ratios under investigation. For example, both for the viscosity ratio of 0.1 and that of 1.1 , at a droplet size of $300 \mu \mathrm{m}$ the critical $C a$ number is increased to approximately the same value as the critical $C a$ number for a droplet size of $100 \mu \mathrm{m}$. This is in contrast with the breakup behavior for which confinement effects can be qualitatively different depending on the viscosity ratio [Vananroye et al. (2006)]. Although the qualitative behavior is the same for the three viscosity ratios, as in bulk conditions, the critical capillary number decreases with increasing viscosity ratio.

\section{B. Critical initial offset for coalescence}

The effect of geometrical confinement on the critical initial offsets for coalescence was already investigated by De Bruyn et al. (2013) for droplet-matrix pairs with a 
viscosity ratio $\lambda=0.1$. It was found that, due to confinement, coalescence is possible up to higher initial offsets. In addition, it was demonstrated that confinement induces a lower critical initial offset, below which coalescence does not occur, because the two droplets exhibit reversing trajectories. Figure 3 displays time sequences of the interaction of two droplets with a diameter $2 R=165 \mu \mathrm{m}$ at $C a$ number 0.004 for three different initial offsets in confined conditions $(H=0.5 \mathrm{~mm})$. These sequences are similar to Fig. 2 of De Bruyn et al. (2013), except that in Fig. 3 the viscosity ratio is 2.6 instead of 0.1 . It can be seen that, similar to systems with viscosity ratio 0.1 , also for a system with viscosity ratio 2.6, a lower critical initial offset exists, below which coalescence does not occur, but instead the two droplets exhibit reversing trajectories. An example of such a reversing trajectory can be seen in Figs. 3(a)-3(e) in which the droplets turn back after their interaction. At intermediate offsets, droplet coalescence occurs, as shown in Figs. 3(f)-3(j), whereas above a critical offset, the droplets rotate over each other without coalescence, as illustrated in Figs. 3(k)-3(o). Figure 4 compares droplet trajectories for systems with viscosity ratios of 0.1 and 2.6 for droplets with size $2 R=165 \mu \mathrm{m}$. Figures 4 (a) and 4(c) display the trajectories in bulk conditions $(2 R / H=0.055)$ and Figs. $4(\mathrm{~b})$ and $4(\mathrm{~d})$ display

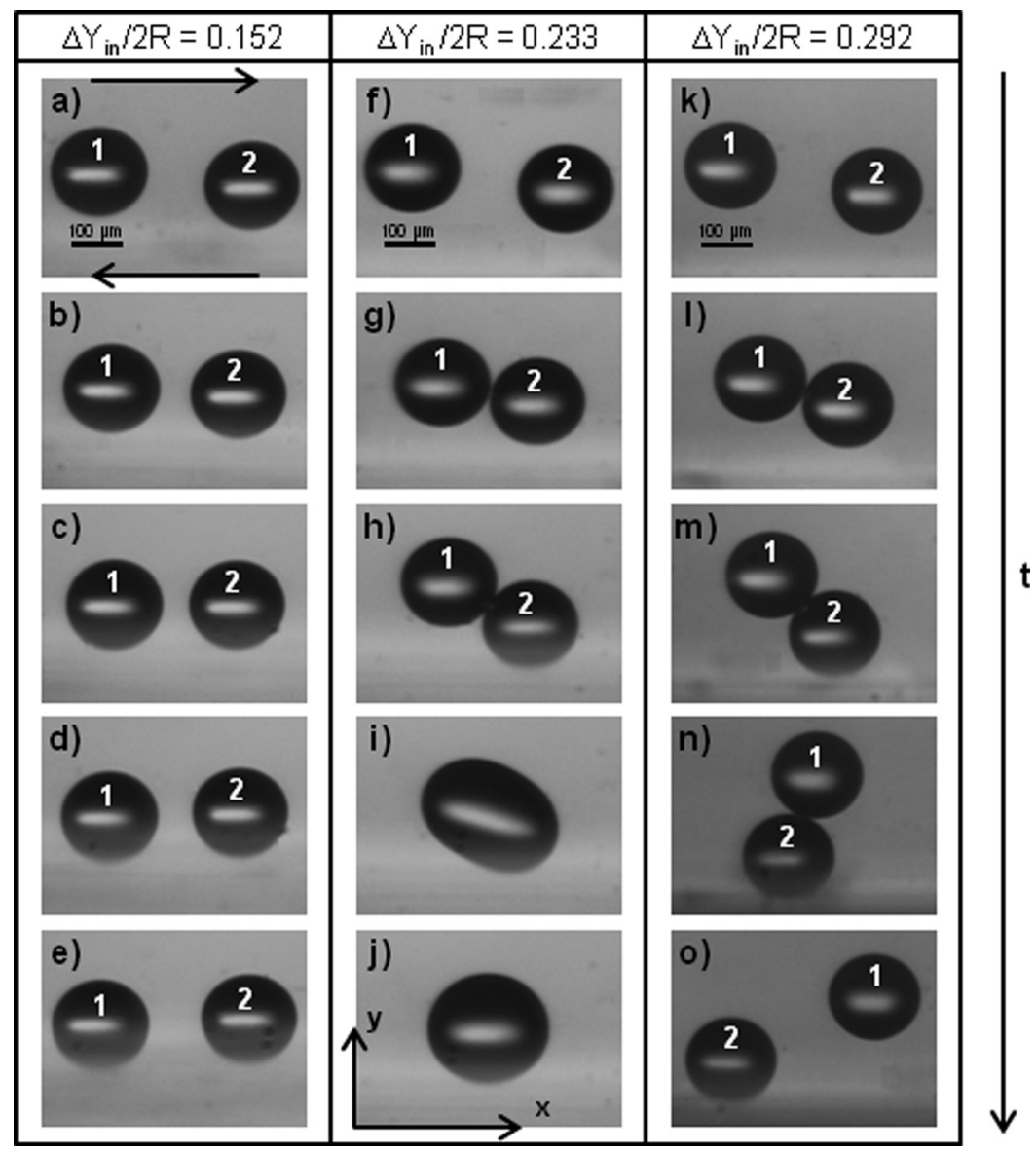

FIG. 3. Time sequence of the interaction of two droplets, $\Delta X_{\text {in }} / 2 R=1.6,2 R=165 \mu \mathrm{m}, H=0.5 \mathrm{~mm}, 2 R /$ $H=0.33, C a=0.004, \lambda=2.6$. (a)-(e) $\Delta Y_{\text {in }} / 2 R=0.152$; (f)-(j) $\Delta Y_{\text {in }} / 2 R=0.233$; and (k) - (o) $\Delta Y_{\text {in }} / 2 R=0.292$. The $x$-direction is the velocity direction, the $\gamma$-direction is the velocity gradient direction. Original images without rescaling of the $x$-axis. 
a) $\lambda=0.1-2 \mathrm{R} / \mathrm{H}=0.055$

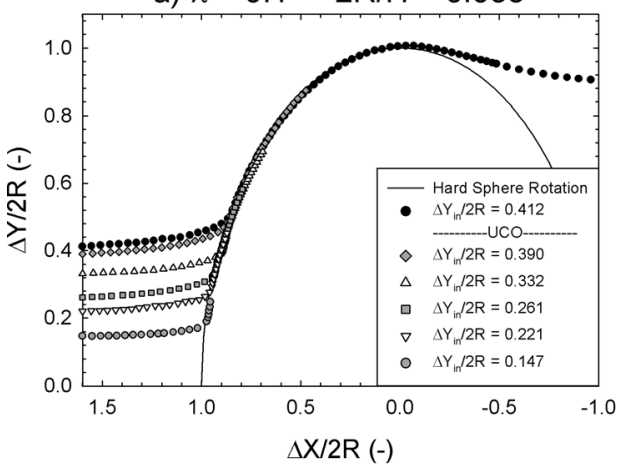

c) $\lambda=2.6-2 \mathrm{R} / \mathrm{H}=0.055$

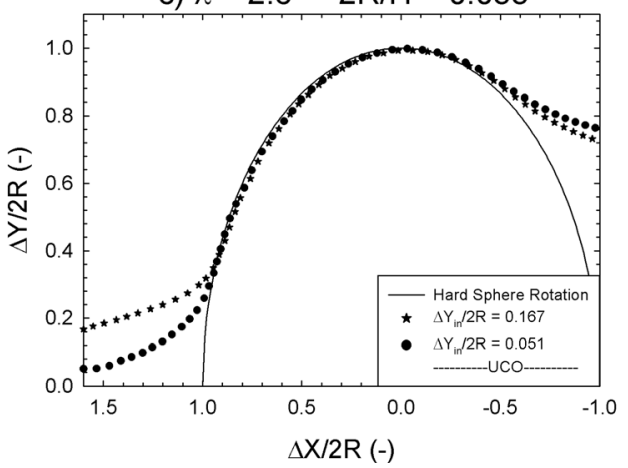

b) $\lambda=0.1-2 \mathrm{R} / \mathrm{H}=0.33$

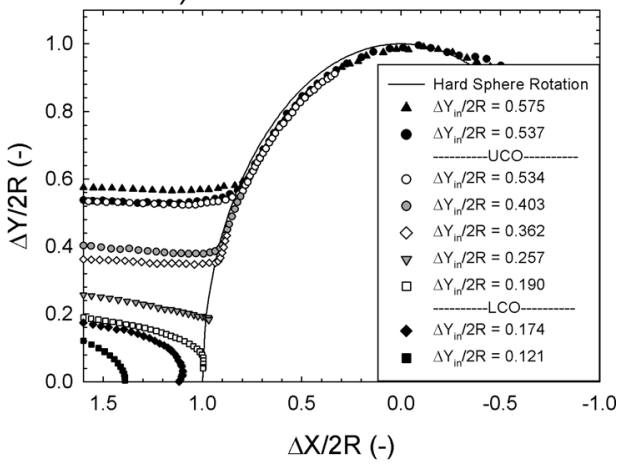

d) $\lambda=2.6-2 \mathrm{R} / \mathrm{H}=0.33$

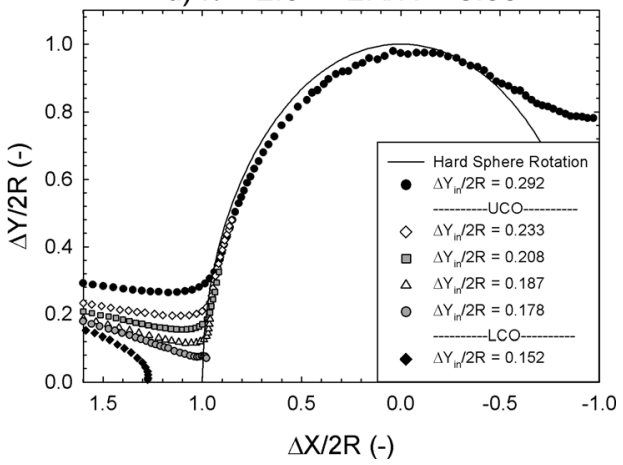

FIG. 4. Evolution of the dimensionless offset $\Delta Y / 2 R$ as a function of dimensionless position $(\Delta X / 2 R)$ for different initial offsets. $\Delta X_{\text {in }} / 2 R=1.6,2 R=165 \mu \mathrm{m}, C a=0.004$. (a) $\lambda=0.1, H=3 \mathrm{~mm}$, (b) $\lambda=0.1, H=0.5 \mathrm{~mm}$, (c) $\lambda=2.6, H=3 \mathrm{~mm}$, and (d) $\lambda=2.6, H=0.5 \mathrm{~mm}$. Filled symbols indicate trajectories for which coalescence does not occur. UCO is the upper critical initial offset and LCO is the lower critical initial offset.

the trajectories in confined conditions $(2 R / H=0.33)$. It must be noted that the trajectories of the three image sequences shown in Fig. 3 can be found in Fig. 4(d). It is clear that for both viscosity ratios the upper critical initial offset, which is the initial offset above which the droplets rotate over each other without coalescence, increases due to confinement. Moreover, it can be seen that for both viscosity ratios, confinement induces a lower boundary for the initial offset, below which the droplets reverse during their interaction and separate without coalescence. Also for droplet-matrix pairs with a viscosity ratio of 1.1 , confinement induces a lower boundary for coalescence and increases the upper boundary for coalescence (results not shown). Hence, it can be concluded that the effects of confinement on the critical offsets for droplet coalescence are similar for the viscosity ratios under investigation.

A quantitative comparison of the upper critical initial offsets in Fig. 4 for the two viscosity ratios reveals that both in bulk and confined conditions, an increase in viscosity ratio substantially decreases the upper critical initial offset. In bulk conditions, the upper critical initial offset at $\lambda=2.6$ is decreased to such a low value that at a $C a$ number of 0.004 , coalescence does not occur anymore. The decrease in the upper critical initial offset with increasing viscosity ratio is in agreement with the decrease in critical $\mathrm{Ca}$ number with increasing viscosity ratio. Both observations are a result from the fact that coalescence is more difficult at higher viscosity ratios. For bulk conditions, this decrease of the upper critical initial offset with increasing viscosity ratio was already reported by 
Yoon et al. (2005) for mixed linear flows. Above the upper critical offset boundary, droplet interactions result in cross-flow migration of the droplets because the offset after interaction is much higher as compared to that before interaction. In confined shear flow, this migration contributes to the formation of layered structures [Pathak et al. (2002)]. Since the upper critical offset boundary and offset after separation depend on the viscosity ratio, it is expected that the occurrence of layered structures and the layer spacing can be tailored by varying the viscosity ratio.

In addition to the upper initial offset boundary, also the lower initial offset boundary, which is only present in confined conditions, can be compared between different viscosity ratios. Figure 5 plots this lower initial offset boundary as a function of the viscosity ratio. The lower boundaries plotted in this figure are measured at a capillary number of 0.010. The trajectories of these measurements will be shown in Fig. 12. It is clear from Fig. 5 that the lower initial offset boundary decreases with increasing viscosity ratio. Hence, for a lower viscosity ratio, the tendency of the droplets to move on reversing trajectories is larger. This observation is in qualitative agreement with the simulation results of Singh and Sarkar (2009), albeit these authors studied reversing trajectories induced by inertia.

It can be concluded that with increasing viscosity ratio both the lower (see Fig. 5) and upper initial offset boundaries (see Fig. 4) decrease. As the decrease of the upper initial offset boundary with viscosity ratio is larger than the decrease of the lower initial offset boundary, the range of initial offsets for which coalescence occurs and thus the coalescence efficiency decreases with viscosity ratio both for bulk and confined conditions. The coalescence efficiencies according to Eq. (7) in De Bruyn et al. (2013) are calculated [coalescence efficiency $\left.=(\text { upper critical initial offset })^{2}-(\text { lower critical initial offset })^{2}\right]$ and the results are summarized in Table II. It is clear that the coalescence efficiency increases due to confinement for both viscosity ratios. Moreover, it is clear that an increase in viscosity ratio results in a decrease of the coalescence efficiency both in bulk and confined conditions. The causes for the effects of viscosity ratio and geometrical confinement on droplet coalescence will be explored in Secs. III D-III F.

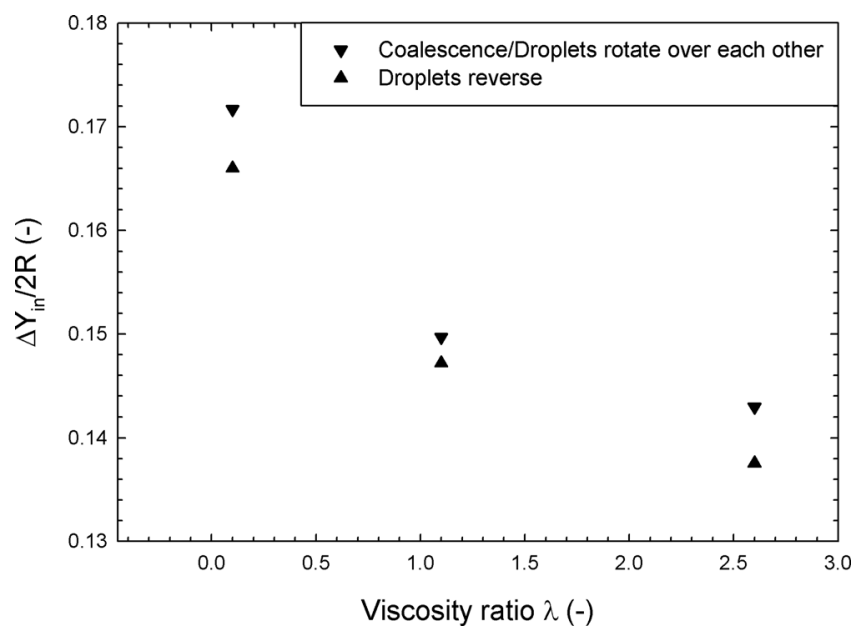

FIG. 5. Lower initial dimensionless offset boundary as a function of viscosity ratio. $\Delta X_{\text {in }} / 2 R=1.6$, $2 R=165 \mu \mathrm{m}, 2 R / H=0.33, C a=0.010$. Upward triangles: largest initial offset for which the two droplets reverse and do not coalesce. Downward triangles: smallest initial offset for which the droplets either coalesce or rotate over each other without coalescence. 
TABLE II. Coalescence efficiencies for viscosity ratios $\lambda=0.1$ and $\lambda=2.6$ both in bulk and confined conditions.

\begin{tabular}{lccccc}
\hline \hline & \multicolumn{2}{c}{$\lambda=0.1$} & & \multicolumn{2}{c}{$\lambda=2.6$} \\
\cline { 2 - 3 } \cline { 5 - 6 } & $H=3 \mathrm{~mm}$ bulk & $H=0.5 \mathrm{~mm}$ confined & & $H=3 \mathrm{~mm}$ bulk & $H=0.5 \mathrm{~mm}$ confined \\
\hline $\mathrm{Ca}=0.002$ & 0.31 & 0.55 & 0.03 & 0.23 \\
$\mathrm{Ca}=0.004$ & 0.16 & 0.25 & & 0.04 \\
\hline \hline
\end{tabular}

\section{Coalescence angles}

The results shown in Secs. III A-III B clearly show that, both in bulk and confined conditions, the parameter space in which coalescence occurs is smaller for systems with a higher viscosity ratio. Apart from the critical conditions for coalescence, also the coalescence angles are investigated for droplet-matrix pairs with different viscosity ratios in bulk and confined conditions. Chen et al. (2009) observed that, for a viscosity ratio $\lambda=1.1$ and a constant initial offset, the coalescence angle increases with the $C a$ number. This increase is partially caused by the higher doublet rotation speed at larger values of $\mathrm{Ca}$ [Allan and Mason (1962)]. In addition, for higher $\mathrm{Ca}$ numbers the hydrodynamic forces are larger, resulting in a larger film drainage area and slower film drainage [Chesters (1991); Yang et al. (2001)]. Chen et al. (2009) also observed that the coalescence angles in confined conditions are lower than the coalescence angles in bulk conditions. To assess the generality of these observations, Fig. 6 plots the coalescence angle as a function of the $C a$ number for a viscosity ratio $\lambda=0.1$. It can be seen that, also for a viscosity ratio smaller than 1 , the coalescence angle indeed increases with the $C a$ number and the coalescence angles in confined conditions are also lower than the coalescence angles in bulk conditions. It can also be seen from Fig. 6 that, at least for a reasonable range of $C a$ numbers under the critical $C a$ number, the difference between the coalescence angles in bulk and confinement is independent of the $C a$ number. Also for a viscosity ratio $\lambda=2.6$, it was observed that the coalescence angles are lower in confined conditions (e.g., for a droplet size of $130 \mu \mathrm{m}$ it was found that for $C a=0.002$ and $\Delta Y_{\text {in }} /$ $2 R=0.23$ the coalescence angle was $56^{\circ}$ for $H=3 \mathrm{~mm}$ and only $51^{\circ}$ for $H=1 \mathrm{~mm}$ ).

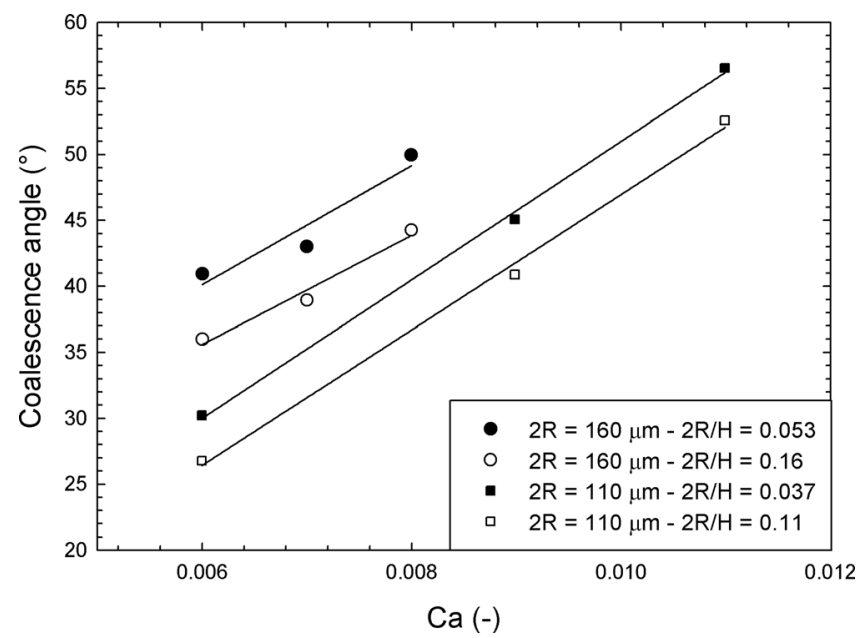

FIG. 6. Coalescence angle as a function of $C a . \lambda=0.1, \Delta X_{\text {in }} / 2 R=1.6$, and $\Delta Y_{\text {in }} / 2 R=0.23$. The filled symbols represent the coalescence angles in the unconfined case, the unfilled symbols represent the coalescence angles for more confined conditions. Lines to guide the eye. 
However, as can be seen in Fig. 2, the $C a$ number range for which coalescence occurs, and thus coalescence angles can be determined, is very limited for this viscosity ratio. Nevertheless, it can be concluded that the effect of confinement on the coalescence angle is similar for all three viscosity ratios.

Since differences in initial offset lead to differences in the gaps between the droplets and the walls, confinement effects on the coalescence angle might be affected by the initial offset. Hence, the coalescence angle as a function of initial offset was investigated for bulk and confined conditions. Figure 7 presents coalescence angles for systems with a viscosity ratio $\lambda=0.1$ as a function of initial dimensionless offset for a droplet diameter $2 R=165 \mu \mathrm{m}$ and a $C a$ number of 0.006 . It can be seen that both for the unconfined and the confined case the coalescence angle increases with increasing initial offset, which is in agreement with literature results for unconfined mixed linear flows [Yoon et al. (2005)]. An increase of the coalescence angle with increasing initial offset is expected because two droplets that start at a larger initial offset come into apparent contact at a larger orientation angle. Hence, by the time film drainage has proceeded enough to allow film rupture, droplet pairs with a larger initial offset will already be at larger orientation angles as compared to droplet pairs with a lower initial offset. It is clear from Fig. 7 that, at a fixed initial offset, the coalescence angle is smaller in the confined case as compared to the unconfined case. This is in agreement with the results of Chen et al. (2009) for a viscosity ratio of 1.1, and with the results shown in Fig. 6, for a viscosity ratio of 0.1 and one specific initial offset. From Fig. 7, it can be concluded that this is valid for the whole range of initial offsets. Moreover, it can be seen that the difference between the coalescence angles in bulk and confinement is approximately the same regardless of the offset. As a consequence of the lower coalescence angle in the confined case, the initial offset needs to exceed a higher value before the droplets rotate over each other without coalescence, which leads to the higher upper initial offset boundary in confinement as shown in Fig. 4. It can be noted that both in bulk and confined conditions, the coalescence angle always remained below $90^{\circ}$. Hence, coalescence in the extensional quadrant of the flow,

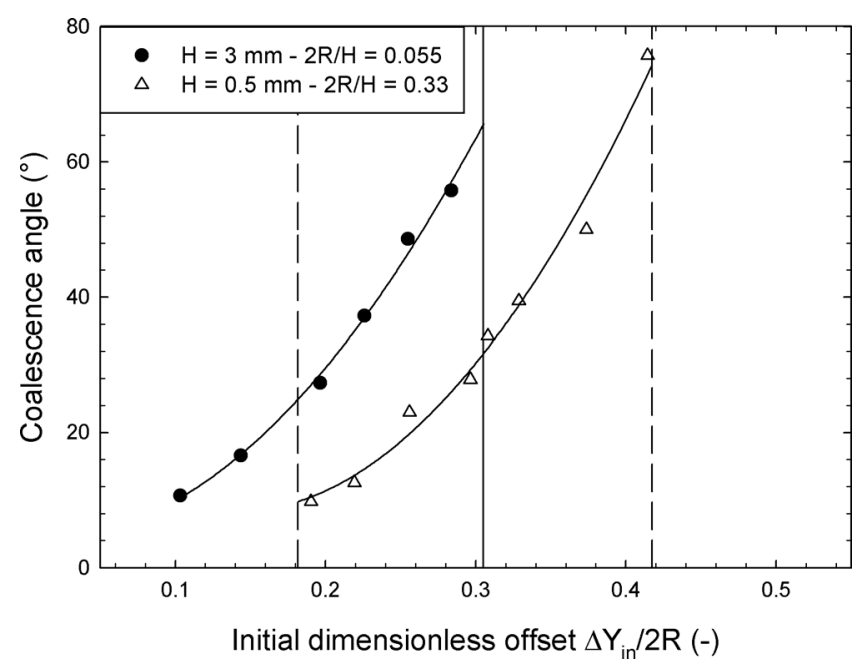

FIG. 7. Coalescence angle as a function of initial dimensionless offset $\Delta Y_{\text {in }} / 2 R . \Delta X_{\text {in }} / 2 R=1.6,2 R=165 \mu \mathrm{m}$, $\lambda=0.1$, and $C a=0.006$. The filled symbols represent coalescence angles in the unconfined case, the unfilled symbols represent coalescence angles in the confined case. Vertical lines depict the initial offset boundaries below which (for the bulk case, solid line) or between which (for the confined case, dashed lines) coalescence occurs. Other lines added to guide the eye. 
which was reported in literature for pure extensional flow [Borrell et al. (2004); Yoon et al. (2005); Bremond et al. (2008)], was never observed here. For the confined dropletmatrix pairs, Fig. 7 also shows the lower boundary for the initial offset, below which there is no coalescence because of the reversal of the droplets. Here, the coalescence angle remained above $0^{\circ}$ for all $\mathrm{Ca}$ numbers and droplet sizes studied. Hence, coalescence again always occurred during the approach of the droplets. A comparison of the initial offset boundaries between bulk and confined conditions for a system with $\lambda=0.1$ and a whole range of $C a$ numbers is presented by De Bruyn et al. (2013). As already shown in Sec. III B, the upper critical offset in bulk conditions is much lower for viscosity ratio $\lambda=2.6$ than for viscosity ratio 0.1 . As a consequence, for droplet-matrix pairs with viscosity ratio $\lambda=2.6$, even for the smallest $C a$ number used in this study $(C a=0.002)$, the range of initial offsets for which there is coalescence both in bulk and confinement is very small. For example, for $C a=0.002$ and $2 R=165 \mu \mathrm{m}$ in bulk conditions coalescence is only possible up to an initial dimensionless offset of 0.17 , which is very close to the initial offset below which the droplets reverse in confinement (see Fig. 5). As a consequence, a comparison of the coalescence angles over a range of initial offsets was not possible for this viscosity ratio.

\section{Droplet trajectories}

In Secs. III A-III C it was clearly shown experimentally that geometrical confinement and viscosity ratio have a substantial effect on several aspects of droplet coalescence such as the coalescence angles, critical $\mathrm{Ca}$ numbers and critical offsets. To obtain more insight in these phenomena, the droplet trajectories have been investigated. First, the effects of confinement are examined. Subsequently, the effects of viscosity ratio will be discussed.

\section{Effects of confinement}

In the previous sections, it has been shown that the coalescence angle is lower in the confined case as compared to the bulk case. As a result of this lower coalescence angle, a larger $C a$ number or a larger initial offset is needed before two droplets rotate over each other without coalescence. This means that, due to confinement, coalescence is promoted for all three viscosity ratios. In order to identify the origin of the lower coalescence angle in confined conditions, the droplet trajectories are studied in detail. Figure 8(a) provides two trajectories which start at the same initial offset, one for bulk conditions at a gap $H$ of $3 \mathrm{~mm}$ and one for confined conditions at a gap $H$ of $0.5 \mathrm{~mm}$. As already seen in Fig. 4, it is clear from Fig. 8(a) that, while in bulk conditions there is a slight increase of the offset during approach of the droplets, in confined conditions the offset decreases during approach. This decrease of the offset during approach can be explained by the presence of recirculation flows at the front and rear of the droplet pair (see further). As a consequence of this decrease in offset during droplet approach, the offset at which the droplets make apparent contact is smaller in the confined case, even though the initial offset is the same as in the unconfined case. Obviously, if the droplets make apparent contact at a smaller offset and hence, at a smaller orientation angle of the droplet doublet, the film drainage can already start at a smaller orientation angle, thereby facilitating droplet coalescence at a smaller angle. However, Fig. 8(a) also shows that, even for droplets that come into apparent contact at the same offset, the confined droplets coalesce at a smaller orientation angle. This implies that due to confinement either the droplet rotation is slowed down or the film drainage is enhanced, even at the same value of the offset.

Figure 8(b) shows the orientation angle of the droplet pair as a function of time for the confined and unconfined collisions shown in Fig. 8(a). This allows for a comparison of 

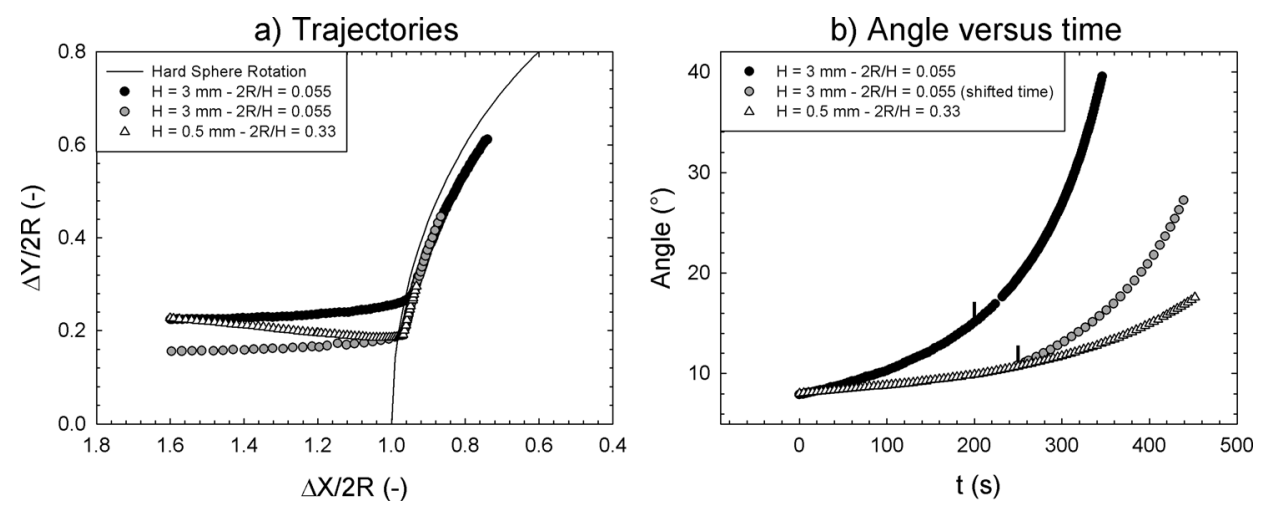

FIG. 8. Comparison of the trajectories for a collision in bulk $(H=3 \mathrm{~mm}, 2 R / H=0.055)$ and a collision in confinement $(H=0.5 \mathrm{~mm}, 2 R / H=0.33)$ with the same initial offset $\left(\Delta Y_{\text {in }} / 2 R=0.225\right)$ and a comparison of the trajectories for a collision in bulk $(H=3 \mathrm{~mm}, 2 R / H=0.055)$ and a collision in confinement $(H=0.5 \mathrm{~mm}, 2 R / H$ $=0.33)$ with the same offset at apparent contact $\left(\Delta Y_{\text {cont }} / 2 R=0.19\right.$ at $\left.d / 2 R=1\right) . \Delta X_{\text {in }} / 2 R=1.6,2 R=165 \mu \mathrm{m}$, $C a=0.008, \lambda=0.1$. (a) Offset $\Delta Y / 2 R$ as a function of $\Delta X / 2 R$. (b) Orientation angle of the droplet pair as a function of time, vertical lines indicate the instants at which $d / 2 R=1, t=0$ corresponds to $\Delta X_{\text {in }} / 2 R=1.6$. For the trajectory in bulk conditions represented by the gray circles the time is shifted so that the time at apparent contact coincides with the time of apparent contact for the trajectory in confined conditions.

the rotation speed of the droplet pairs in bulk and confined conditions. The starting point at time $t=0 \mathrm{~s}$ is the time at which $\Delta X / 2 R=1.6$. The instant at which $d / 2 R=1$, which is an indication for the onset of film drainage, is marked by vertical lines. It is clear from Fig. 8(b) that, in confined conditions, due to the decrease in offset during the approach of the droplets, the increase in the orientation angle with time is much slower as compared to bulk conditions. This is expected because, even in the case when there would be no hydrodynamic interaction between the two droplets, the relative velocity in the $x$-direction between the two droplets is proportional to $\Delta Y$. Also after apparent contact, the droplet pair in confined conditions rotates much slower than that in unconfined conditions due to the lower offset. This can be explained by the fact that the rotation speed increases with the doublet orientation angle up to an angle of $90^{\circ}$ [Allan and Mason (1962)]. Hence, in confined conditions there is more time for film drainage, which further contributes to a reduction of the coalescence angle as compared to bulk conditions. Figure 8(b) also compares the rotation speeds between an unconfined and a confined droplet pair with the same offset at apparent contact. Thereto, the time for the collision in bulk conditions (gray circles) is shifted so that the time of apparent contact coincides with the time of apparent contact for the collision in confined conditions. It is clear from Fig. 8(b) that in confined conditions the droplets rotate slower than in bulk conditions even if they make apparent contact at the same offset. This difference between the confined and unconfined collisions can be explained by the flow fields around the droplet pair (see further). As a consequence of the slower rotation, the orientation angle of the droplet pair at which coalescence occurs, is lower in confined conditions, even when the droplets make apparent contact at the same offset. Figure 9 shows the coalescence angle as a function of the offset at apparent contact. This figure confirms that the coalescence angle is lower due to confinement, even for droplet pairs for which the offset at apparent contact is the same. Moreover, it can also be seen that the offset at apparent contact up to which coalescence occurs is larger in confined conditions. A comparison of the drainage times between bulk and confinement in Fig. 8(b) reveals that the drainage times are approximately the same. According to Borrell et al. (2004), the drainage process is dominated by the time history of the force along the line of centers of the droplet pair. Due to the 


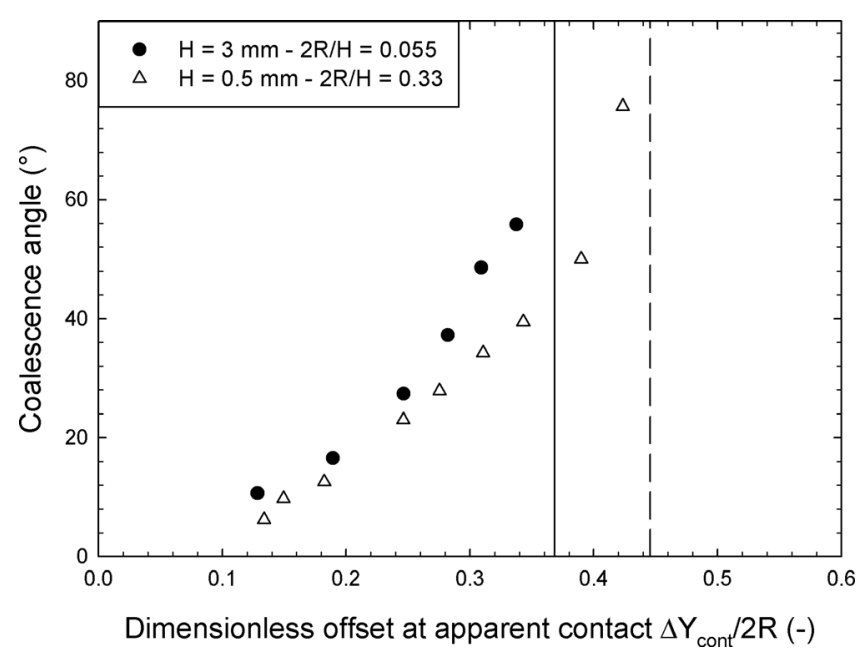

FIG. 9. Coalescence angle as a function of the dimensionless offset at apparent contact $\Delta Y_{\text {cont }} / 2 R . \Delta X_{\text {in }} / 2 R$ $=1.6,2 R=165 \mu \mathrm{m}, \lambda=0.1$, and $C a=0.006$. Vertical lines depict the offset boundaries at contact below which coalescence occurs for bulk conditions (solid line) and confined conditions (dashed line).

smaller rotation speed in confined conditions in comparison to bulk conditions, a droplet pair in confined conditions spends more time at lower orientation angles. In bulk conditions the hydrodynamic force, pushing the droplets together, reaches a maximum at an orientation angle $\theta$ of $45^{\circ}$, which is equivalent to a dimensionless offset of $\Delta Y / 2 R=\sin$ $45^{\circ}=0.707$ [Batchelor and Green (1972); Zinchenko (1978); Jaeger et al. (1994)]. In confined conditions, this maximum occurs even at a larger orientation angle [Chen et al. (2009)]. Hence, at first sight it could be expected that the force pushing the two droplets together is smaller in confined conditions in comparison to bulk conditions. However, at the same orientation angle, the hydrodynamic force might also be affected by confinement, an aspect which is discussed in Sec. III F.

\section{Effects of viscosity ratio}

Besides the effects of confinement, in Secs. III A-III C, also the effect of viscosity ratio has been investigated systematically. In Secs. III A and III B, it was reported that the critical $\mathrm{Ca}$ number and the upper critical initial offset decrease with increasing viscosity ratio. A first reason for this is the lower mobility of the interface at the higher viscosity ratio, which hinders film drainage [Chesters (1991); Mousa et al. (2002)]. However, a comparison of Fig. 4(a) with Fig. 4(c) learns that also the droplet trajectories are different for different viscosity ratios. To clarify this further, Fig. 10(a) compares two trajectories in bulk conditions with the same initial offset but with a different viscosity ratio. It is clear from Fig. 10(a) that for the higher viscosity ratio the offset increases more during approach of the droplets. This implies that hydrodynamic interactions between droplets are more pronounced in systems with a high viscosity ratio as compared to systems with a low viscosity ratio, which is in agreement with the observations of Tretheway et al. (1999) in extensional flow. As a consequence of the stronger hydrodynamic interactions, for the higher viscosity ratio the droplets make apparent contact at a higher offset and consequently a larger orientation angle, thus providing a second cause for the suppression of coalescence with increasing viscosity ratio.

Finally, Fig. 10(b) plots the orientation angle of the droplet pair as a function of time corresponding to the two trajectories shown in Fig. 10(a). The starting point of each 
a) Trajectories

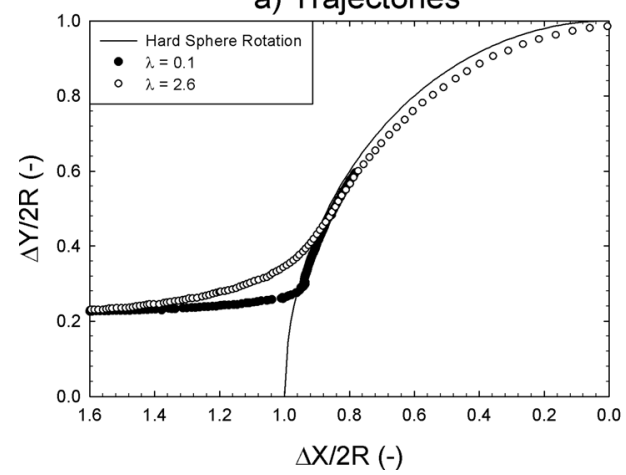

b) Angle versus time

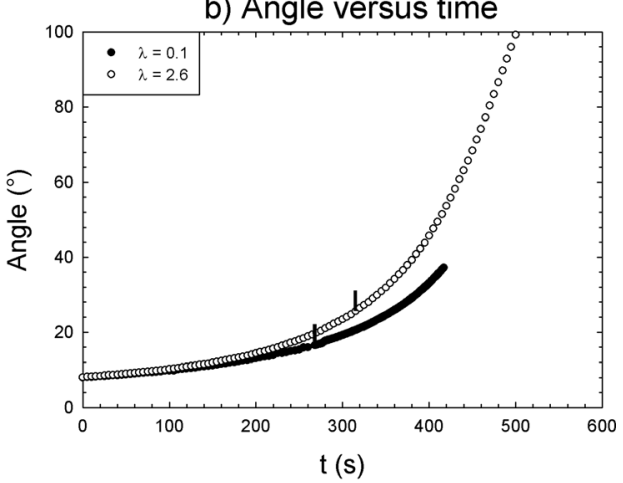

FIG. 10. Comparison of the trajectories for a collision with viscosity ratio $\lambda=0.1$ and a collision with viscosity ratio $\lambda=2.6$. Both collisions are in bulk conditions and have the same initial offset. $\Delta Y_{\text {in }} / 2 R=0.229$, $\Delta X_{\text {in }} / 2 R=1.6, H=3 \mathrm{~mm}, 2 R=165 \mu \mathrm{m}$, and $C a=0.006$. (a) Offset $\Delta Y / 2 R$ as a function of $\Delta X / 2 R$ and (b) orientation angle of the droplet pair as a function of time, vertical lines indicate the instants at which $d / 2 R=1, t=0$ corresponds to $\Delta X_{\mathrm{in}} / 2 R=1.6$.

curve, which is the point at time $t=0 \mathrm{~s}$, is the time at which $\Delta X / 2 R=1.6$. The instant at which $d / 2 R=1$, which is an indication for the onset of film drainage, is marked by vertical lines. It can be seen from Fig. 10(b) that for the higher viscosity ratio, due to the larger increase of the offset during approach of the droplets, the increase in the orientation angle with time is faster as compared to that for the lower viscosity ratio system.

For a better understanding of the lower initial offset boundaries shown in Fig. 5, droplet trajectories for initial offsets close to the lower initial offset are compared in Fig. 11 for viscosity ratio 0.1 and 2.6. For offsets below the lower critical offset boundary, reversing trajectories occur, which have been attributed to the presence of reversing streamlines at the front and rear of confined droplet pairs [De Bruyn et al. (2013)]. It can be seen that the shape of the trajectories is different for the different viscosity ratios. In Fig. 11(b), one trajectory for viscosity ratio 0.1 is added to allow for a direct comparison of the shape of the trajectory between viscosity ratios 0.1 and 2.6. This figure shows that during the first stage of the approach $(\Delta X / 2 R=1.6$ till approximately 1.2$)$, the decrease in offset is slightly larger for the higher viscosity ratio. However, when the droplets approach each other closer $(\Delta X / 2 R \leq 1.2)$, there is an inflection in the curve for the larger

(a) $\lambda=0.1$

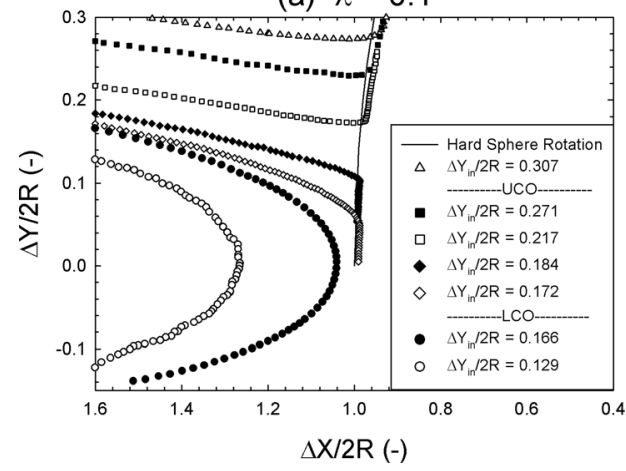

(b) $\lambda=2.6$

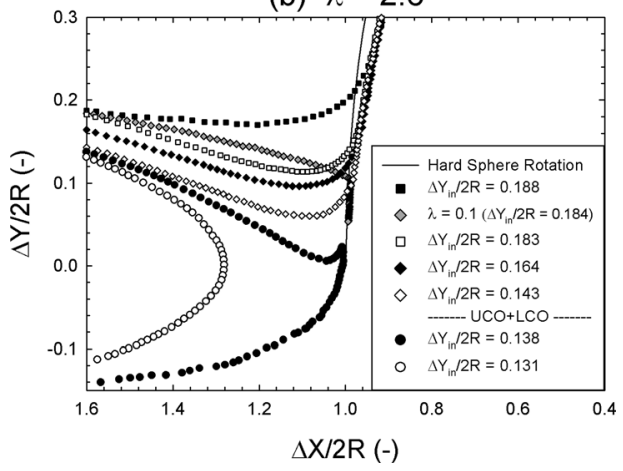

FIG. 11. Evolution of the vertical offset $\Delta Y / 2 R$ as a function of position $(\Delta X / 2 R)$ for different initial offsets around the lower critical offset boundary. $\Delta X_{\text {in }} / 2 R=1.6,2 R=165 \mu \mathrm{m}, H=0.5 \mathrm{~mm}, 2 R / H=0.33$, and $C a=0.010$. (a) $\lambda=0.1$ and (b) $\lambda=2.6$ and one trajectory for $\lambda=0.1$ at $\Delta Y_{\text {in }} / 2 R \sim 0.184$. 
viscosity ratio, i.e., the trajectory goes from slightly concave downward (negative second derivate) to concave upward (positive second derivate). When the droplets approach even closer $(\Delta X / 2 R \leq 1.1)$, the offset goes through a minimum and then starts increasing. For the viscosity ratio of 0.1 [Fig. 11(a)], on the other hand, the curves remain concave downward almost up to the point where the two droplets make apparent contact. Then, the droplet trajectory abruptly changes direction when the droplets make apparent contact. It can also be noted that for the viscosity ratio of 0.1 , there is a range of initial offsets above the lower critical offset, for which the droplets start rotating in the direction of the reversing streamlines after apparent contact and subsequently coalesce. On the other hand, it can be seen from Fig. 11(b) that for the higher viscosity ratio, most droplets that undergo reversing streamlines, remain at a rather large separation distance. The decrease of the offset range in which reversing trajectories occur with increasing viscosity ratio (see Fig. 5) is thus caused by the fact that droplets with a larger viscosity ratio have a greater tendency to being pulled along with the clockwise rotation of the fluid in the second droplet.

Numerical simulations in 2D of the approach and interaction of two droplets under the same conditions as that of the experimental trajectories of Fig. 11 were performed. Figure 12 plots the simulated trajectories in confined conditions for viscosity ratios 0.1 and 2.6. The simulations confirm that, when starting at the same initial offset, the offset at apparent contact increases with viscosity ratio (e.g., see trajectories for $\Delta Y_{\text {in }} / 2 R$ $=0.30$ ). In addition, it is clear from Fig. 12 that the lower initial offset boundary, below which the droplets reverse flow direction, is larger for the smaller viscosity ratio. This is in agreement with the results in Fig. 5. As for the experimental trajectories, it can also

a) Trajectories $\lambda=0.1$

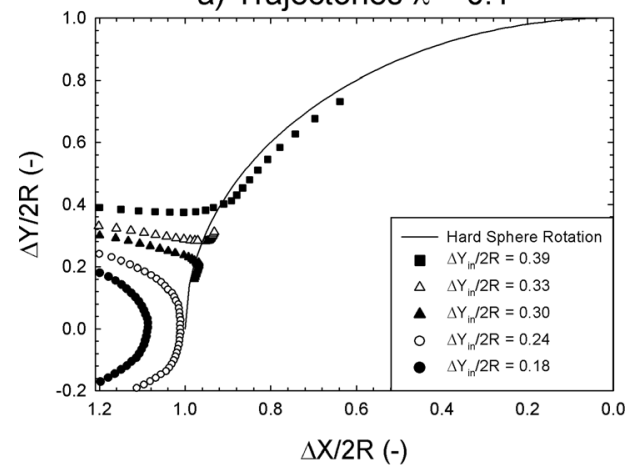

c) Streamlines $\lambda=0.1$

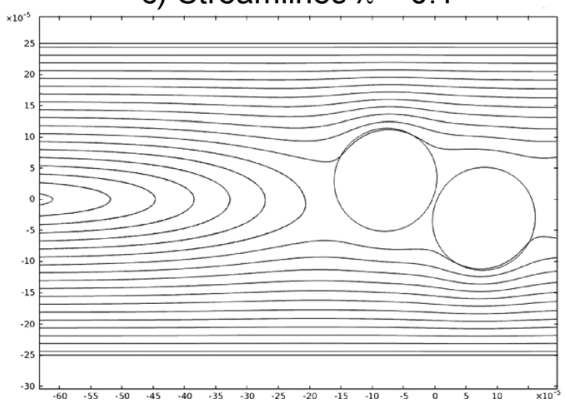

b) Trajectories $\lambda=2.6$

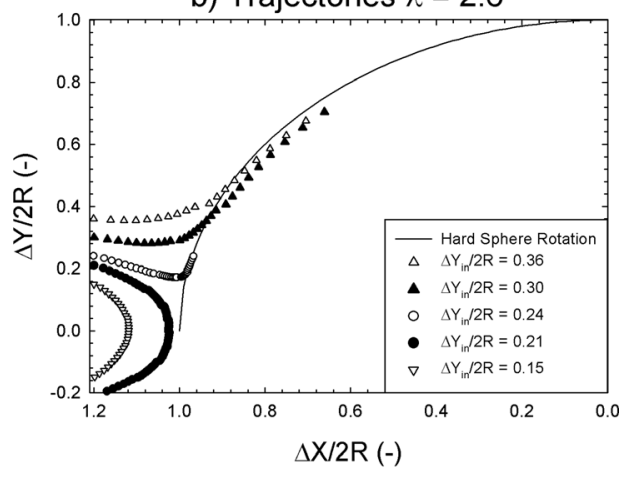

d) Streamlines $\lambda=2.6$

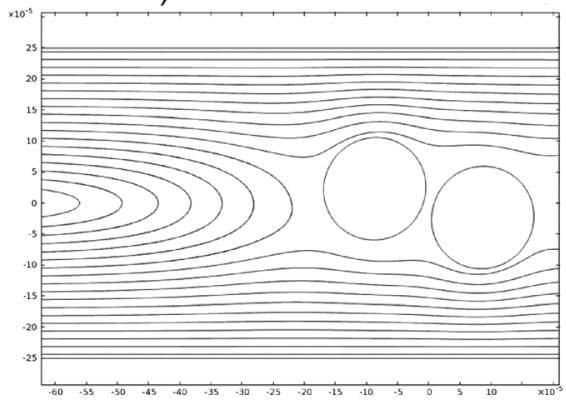

FIG. 12. (a) and (b) Simulated droplet trajectories for different initial offsets. $\Delta X_{\text {in }} / 2 R=1.2,2 R=165 \mu \mathrm{m}$, $H=0.5 \mathrm{~mm}, 2 R / H=0.33, C a=0.010$. (a) $\lambda=0.1$ and (b) $\lambda=2.6$. After apparent contact the simulations are stopped. Coalescence is not included in the graphs. (c) and (d) Simulation in $2 \mathrm{D}$ of the streamlines around two colliding droplets. $2 R=165 \mu \mathrm{m}, H=0.5 \mathrm{~mm}, 2 R / H=0.33, C a=0.010, \Delta Y / 2 R=0.4$. (c) $\lambda=0.1$ and (d) $\lambda=2.6$. 
be seen that, for the higher viscosity ratio, the droplet trajectory turns upward at a larger separation distance between the droplets. This can, e.g., be seen from a comparison between the curves for $\Delta Y_{\text {in }} / 2 R=0.33$ in Fig. 12(a) and $\Delta Y_{\text {in }} / 2 R=0.30$ in Fig. 12(b). On the other hand, for the smaller viscosity ratio, the transition between approach of the droplets and rotation over each other occurs almost discontinuously at the moment of droplet contact, at least, if the initial offset is not too large. It must be noted that coalescence can not be predicted with the present simulations. The last point of the trajectories thus merely corresponds to the end of the simulation run and does not indicate coalescence.

Figures 12(c) and 12(d) provide the streamlines around a droplet pair with $\Delta Y$ / $2 R=0.40$ for systems with a viscosity ratio $\lambda$ of, respectively, 0.1 and 2.6 in confined conditions. These figures show that large recirculation zones are present at the front and rear of the confined droplet pair, which are absent in bulk conditions. While in bulk conditions all streamlines pull the upper left droplet over the lower right droplet, in confined conditions there is a competition between streamlines pulling the upper left droplet over the lower right droplet and streamlines of the recirculation zone pulling the upper left droplet down. Hence, the second droplet will not easily rotate over the first one but remains trapped in this recirculation zone which causes the slower rotation of the droplet doublet in confinement, as observed in Fig. 8. These figures also clearly show that the recirculation zone at the front and rear of the droplet pair is rather insensitive to viscosity ratio. The larger tendency of droplets with a high viscosity ratio to rotate over each other in the direction of the shear is thus most probably caused by the stronger tangential lubrication force between the droplets. Simulations indeed showed that the tangential stress along the droplet interface is significantly larger for the system with viscosity ratio 2.6. The simulations thus confirm the experimental results and allow to shed light on the effect of viscosity ratio on the lower offset boundary.

\section{E. Mastercurves for droplet trajectories}

It was shown in Fig. 8 of Sec. III D that geometrical confinement substantially affects the doublet rotation speed, even if the droplets make apparent contact at the same offset. An additional aspect of interest is whether these confinement effects depend on the initial offset of the droplets or change in magnitude during the droplet interaction. To assess these questions, the kinetics of doublet rotation was investigated for droplet pairs with different initial offsets. Figures 13(a) and 13(b) plot the doublet orientation angle as a function of shifted time for different collisions, starting from the instant at which the two droplets make apparent contact $(d / 2 R=1)$, respectively, for a gap $H=3 \mathrm{~mm}$ and a gap $H=0.5 \mathrm{~mm}$. For all collisions the $C a$ number is 0.008 and the droplet size $2 R=165 \mu \mathrm{m}$, whereas the initial offset was varied. The original curves (starting from $\Delta X_{\text {in }} / 2 R=1.6$ ) are shown in the insets in Fig. 13. Subsequently, the curves were shifted horizontally with a time $t^{*}$, resulting in Figs. 13(a) and 13(b). For each curve, the shift factor $t^{*}$ is determined as such that the doublet orientation angle $\theta$ is $90^{\circ}$ at time $t-t^{*}=0$. It can be seen that, for both confinement ratios, all curves of orientation angle versus shifted time collapse onto a master curve. This implies that once the two droplets are in apparent contact, the rotation speed does not depend on the approach history of the droplets but only on the current orientation angle of the doublet. Also for other $\mathrm{Ca}$ numbers in the range for which the droplet shapes remain approximately spherical, similar master curves could be constructed (results not shown). Bartok and Mason (1957) have experimentally verified that the rotation speed of a pair of rigid spheres in shear flow equals the rotation speed of a rigid prolate spheroid of axis ratio 2, which can be described by the equations 
a) $2 \mathrm{R} / \mathrm{H}=0.055$

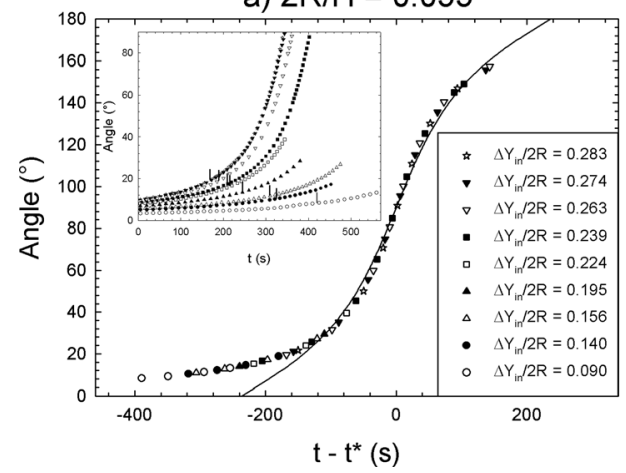

b) $2 \mathrm{R} / \mathrm{H}=0.33$

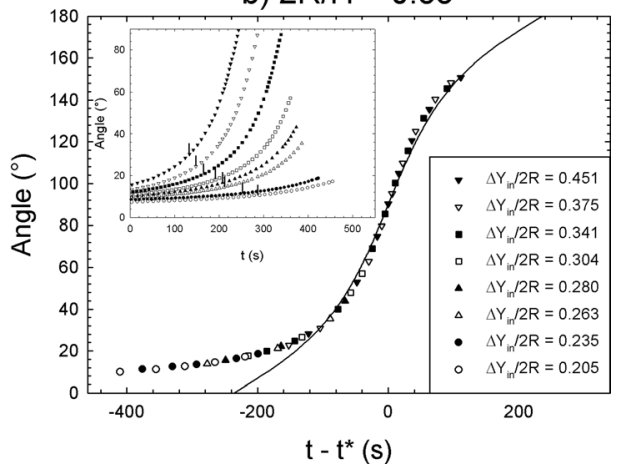

FIG. 13. Orientation angle of the droplet pair as a function of shifted time, starting from the instant at which $d / 2 R=1$. The time for all the collisions is shifted with $t^{*} . \Delta X_{\mathrm{in}} / 2 R=1.6,2 R=165 \mu \mathrm{m}, C a=0.008, \lambda=0.1$. (a) $H=3 \mathrm{~mm}, 2 R / H=0.055$ and (b) $H=0.5 \mathrm{~mm}, 2 R / H=0.33$. The solid line is the rotation speed according to Eq. (6). The insets show the orientation angle of the droplet pair as a function of the original time, where $t=0$ corresponds to $\Delta X_{\text {in }} / 2 R=1.6$. Vertical lines indicate the instants at which $d / 2 R=1$.

given by Jeffery (1922). According to these equations, the orientation angle of a spheroid as a function of time can be calculated from the following equation:

$$
\tan \left(\theta-90^{\circ}\right)=2 \tan \left(\frac{2 t \dot{\gamma}}{5}\right) .
$$

It is clear that this equation does not predict any dependency on the approach history but it predicts a rotation speed which is only dependent on the current orientation angle and the shear rate, which is expected in Stokes flow conditions. The rotation speed according to Eq. (6) is also plotted in Fig. 13. It can be seen that both for bulk and confined conditions the rotation speed of the droplet pair becomes equal to the rotation speed of a hard sphere rotation starting from an orientation angle of around $30^{\circ}$, which is equivalent to a dimensionless offset $\Delta Y / 2 R=\sin \left(30^{\circ}\right)=0.5$. At lower orientation angles the rotation speed of the droplet pair is lower.

Figure 14 compares the master curves from Fig. 13 for the two confinement ratios. It is clear from this figure that below an orientation angle of around $30^{\circ}$, the confined droplets rotate slower than the unconfined ones. This confirms the lower rotation speed due to confinement which was already seen in Fig. 8(b). It must be noted, however, that this lower rotation speed due to confinement is only retained for a limited time after apparent contact $(d / 2 R=1)$. When the orientation angle reaches $30^{\circ}$, the rotation speed becomes equal to the rotation speed of a pair of hard spheres in bulk conditions, at least for confinement ratios $2 R / H$ up to 0.33 . Hence, above an orientation angle of $30^{\circ}$, which is equivalent to a dimensionless offset of 0.5 , the available contact time is not affected by confinement, at least for confinement ratios $2 R / H$ up to 0.33 . For the systems with higher viscosity ratios, master curves were also constructed (results not shown). These curves also follow Eq. (6) at large orientation angles and show similar effects of confinement as for the system with $\lambda=0.1$ at low orientation angles.

\section{F. Forces on droplet pairs in shear flow}

From a comparison between a glancing collision and a head-on collision with a timedependent force mimicking the force history due to rotation of the droplets in the glancing collision, Borrell et al. (2004) concluded that the drainage process is dominated by 


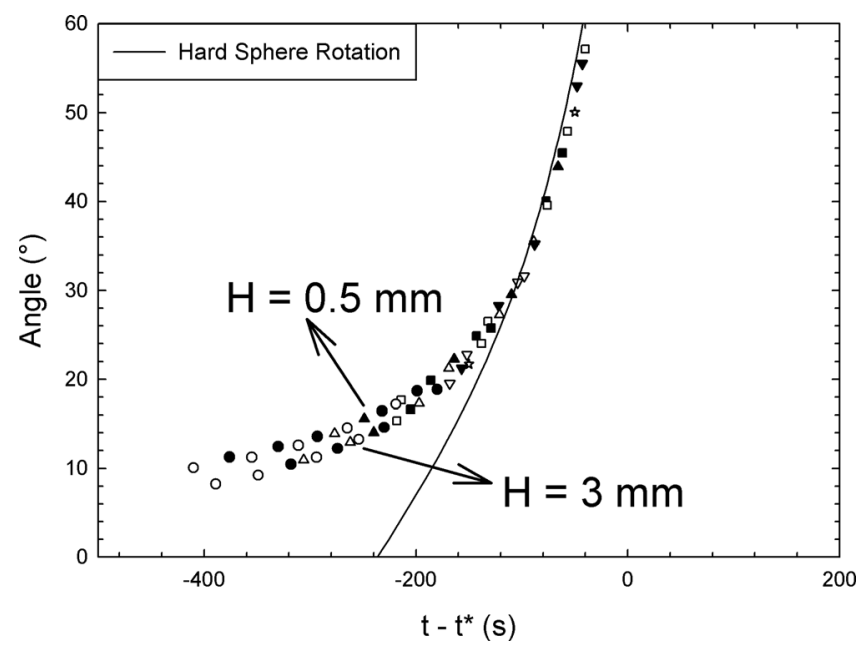

FIG. 14. Orientation angle of the droplet pair as a function of shifted time, starting from the instant at which $d / 2 R=1$. The time for all the collisions is shifted with $t^{*} . \Delta X_{\text {in }} / 2 R=1.6,2 R=165 \mu \mathrm{m}, C a=0.008, \lambda=0.1$. Upper master curve is for $H=0.5 \mathrm{~mm}$, lower master curve is for $H=3 \mathrm{~mm}$.

the time history of the force along the line of centers of the droplet pair. In bulk conditions, the hydrodynamic force $F_{h}$ along the line of centers of a droplet pair in shear flow can be described by the following expression [Jaeger et al. (1994)]:

$$
F_{h}=4.34\left(\frac{2 / 3+\lambda}{1+\lambda}\right) \pi \cdot \eta_{m} \cdot \dot{\gamma} \cdot R^{2} \cdot \sin \left(2\left(90^{\circ}-\theta\right)\right) .
$$

Hence, the hydrodynamic force along the line of centers is dependent on the orientation angle of the droplet pair. The force is zero at an orientation angle of $0^{\circ}$, increases to a maximum at an orientation angle of $45^{\circ}$ and then decreases again to become zero at an orientation angle of $90^{\circ}$, after which the droplets are being pulled apart. In Sec. III D (discussion of Fig. 8), it was already seen that confinement changes the angle at apparent contact and the rotation speed of the droplet doublet and thereby affects the time history of the hydrodynamic force.

The question that still remains is whether there is a difference in the hydrodynamic forces for an unconfined and a confined droplet pair at the same orientation angle. Using analytical results for single droplets between parallel plates, Chen et al. (2009) already argued that due to confinement extra hydrodynamic wall forces arise that can have the same order of magnitude as the hydrodynamic forces originating from bulk shear flow and, hence, can influence the dynamics of film drainage, especially at higher orientation angles. In this paper, 2D finite element simulations will be used to estimate the hydrodynamic stresses on a droplet in a droplet doublet. The normal component of the hydrodynamic stress plotted as a function of the position on the droplet interface, expressed by the angle $\alpha$, is presented in Figs. 15(a) and 15(b) for pairs of touching droplets with, respectively, a high and a low orientation angle. Representative normalized vector plots of the viscous stress for a low and a high orientation angle are provided as insets in Fig. 15. From Fig. 15, it can be seen that in the region opposite to the second droplet, approximately for $\alpha$ from $90^{\circ}$ to $180^{\circ}$, there is compression. In the neighboring regions, approximately from $0^{\circ}$ to $90^{\circ}$ and from $180^{\circ}$ to $270^{\circ}$, the droplet experiences a tensile stress. In the region where the two droplets touch each other, the droplet interfaces are very close 
a) $\theta=50^{\circ}$

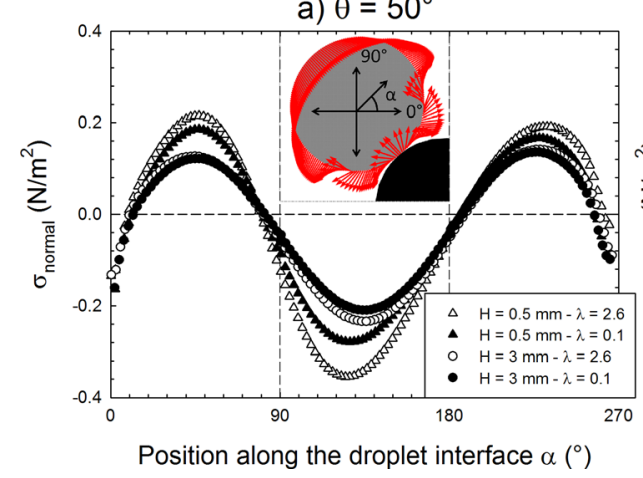

b) $\theta=6^{\circ}$

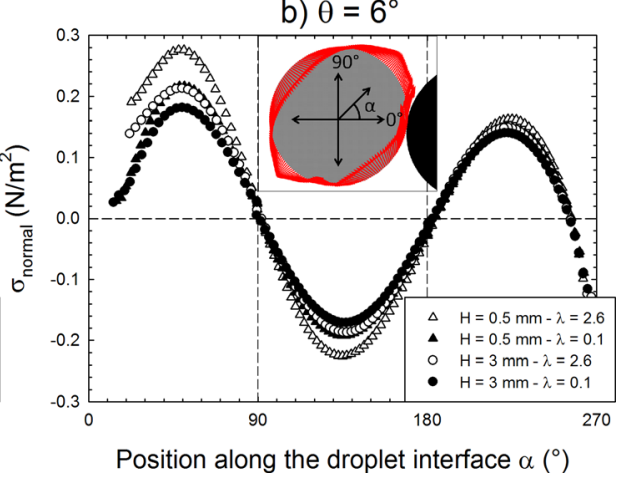

FIG. 15. Normal stress on the droplet interface of a droplet in a droplet pair as a function of the position along the droplet interface. $2 R=165 \mu \mathrm{m}, C a=0.008$. (a) Doublet orientation angle $\theta=50^{\circ}$. (b) Doublet orientation angle $\theta=6^{\circ}$. The insets show the simulated stress vectors on the droplet interface for a droplet pair in shear flow with a gap height $H=3 \mathrm{~mm}$.

together. For an accurate simulation of the stresses in this region, the size of the mesh should be much smaller than the separation distance between the two droplets. This was, however, not practically feasible in the present simulations. Therefore, the simulated stresses in the contact region are not reliable and were thus not included in Fig. 15. Film drainage is governed by the compression force along the line of centers of the droplet, which can be obtained as the integral of the normal and shear stresses along the interface, projected along the line of centers of the droplets. Hence, the values of the compressional stress for $\alpha$ between $90^{\circ}$ and $180^{\circ}$ are the most relevant to estimate confinement effects. Figure 15 shows that for both viscosity ratios and irrespective of the orientation angle, confinement causes an increase of the compressional stress. The difference in hydrodynamic stresses between bulk and confined conditions becomes less pronounced for low orientation angles of the droplet doublet. This confirms the argumentation of Chen et al. (2009) that due to confinement extra hydrodynamic wall forces arise, which have the same order of magnitude as the hydrodynamic forces originating from bulk shear flow and thus can influence the dynamics of film drainage. It should be mentioned here that the stress values presented in Fig. 15 are the results of a 2D simulation which most probably does not fully capture the 3D dynamics of interacting droplets. Hence, the values of the normal stress obtained with this simulation will not be quantitatively the same as those in the experimental system. Nevertheless, Fig. 15 clearly shows the qualitative effects of geometrical confinement on the stresses on interacting droplets.

According to Yang et al. (2001), the effect of a higher hydrodynamic force on the film drainage process depends on the film shape. If the droplets remain spherical during the film drainage process, an increase of the hydrodynamic force will result in faster film drainage. However, if the hydrodynamic force becomes too large, the pressure between the droplet interfaces can reach the Laplace pressure and the droplet interfaces will flatten. The lateral dimension of the thin film can be estimated by equating the hydrodynamic force to the product of the film drainage area with the Laplace pressure, which is of $O(\Gamma / R)$. Hence, a rough estimation of the radius of the film a can be found [Yang et al. (2001)],

$$
a^{2} \sim \frac{\mathrm{R}}{\Gamma} \cdot \mathrm{F}
$$


with $F$ the hydrodynamic force on the droplet doublet. When substituting this expression in the equation for the thinning rate of two squeezing disks [Eq. (19) in Yang et al. (2001)], it becomes clear that the film drainage rate scales as

$$
\frac{1}{h^{2}} \frac{d h}{d t} \sim-\frac{\Gamma^{3 / 2}}{\eta_{d} R^{3 / 2} F^{1 / 2}} .
$$

In this case, the film drainage will thus be slower if the hydrodynamic force increases. This is because a larger force will result in a larger flattened film that needs to be drained. In the Appendix it is argued that in most of the cases investigated here, the droplets are flattened during the largest part of their interaction and the additional hydrodynamic wall forces will thus hinder coalescence, thereby partially counteracting the coalescence promotion caused by the differences in droplet trajectories and doublet rotation speeds.

In conclusion, the effects of geometrical confinement on droplet coalescence are generated by a complex interplay of several factors that may counteract each other. Hence, sophisticated 3D numerical simulations are needed to unravel these effects and to predict the outcomes of droplet collisions in confined shear flow. However, up to now simulating droplet coalescence in shear flow still remains challenging and matching the experimental conditions is often computationally impractical [Shardt et al. (2013)].

\section{CONCLUSIONS}

The effect of geometrical confinement on droplet coalescence in shear flow is systematically investigated for different viscosity ratios $(\lambda=0.1,1.1$, and 2.6$)$. The main conclusion is that the effects of confinement are similar for all three viscosity ratios. This is in contrast with droplet breakup, for which confinement effects can be qualitatively different, depending on the viscosity ratio [Vananroye et al. (2006)]. Above a confinement ratio of $2 R / H \approx 0.2$, the critical capillary number increases with confinement ratio for the three viscosity ratios. Furthermore, for all three viscosity ratios, confinement makes coalescence possible up to higher initial offsets. On the other hand, confinement also induces a lower boundary for the initial offset below which the offset decreases to zero during approach of the droplets after which the droplets reverse without coalescence. Finally, for all initial offsets confinement decreases the coalescence angle.

The differences between bulk and confined droplet pairs can be explained by a complex interplay of changes in the flow field and additional wall forces induced by confinement. Confinement induces large recirculation zones at the front and the rear of the droplet pair. As a consequence of these recirculation zones the offset decreases during approach of the droplets. For low initial offsets, this can lead to reversing trajectories, which cause the lower boundary for the initial offset. A second consequence of this decrease of the offset during approach is that the offset at apparent contact is lower in confined conditions as compared to bulk conditions. Hence, film drainage can already start at a lower angle. Moreover, it is observed that in confined conditions, the rotation speed is lower in comparison with bulk conditions, which results in a larger contact time available for film drainage. Finally, as a consequence of the lower offset at apparent contact and lower rotation speed in confinement, the droplet pair spends more time at lower coalescence angles where the force pushing the droplets together is smaller. Master curves of the orientation angles versus time could be constructed for droplet pairs with different initial offsets. This demonstrated that, once the doublet orientation angle exceeds $30^{\circ}$, the rotation kinetics becomes independent of confinement, viscosity ratio, 
and approach history. Nevertheless, in confinement additional hydrodynamic wall forces are present during the complete interaction process.

Although the qualitative effects of confinement are similar for the different viscosity ratios, there is a quantitative difference between the viscosity ratios. With increasing viscosity ratio, coalescence becomes more difficult. This results in lower critical $\mathrm{Ca}$ numbers and lower upper initial offset boundaries for the higher viscosity ratios. The reason for this more difficult coalescence is twofold. First, for a higher viscosity ratio the mobility of the interface is lower, which results in a more difficult film drainage. Second, for the higher viscosity ratios the two droplets make apparent contact at a higher offset. In addition, the lower initial offset boundary, below which the droplets reverse, decreases with viscosity ratio. This is most probably caused by the larger tangential stress present at the droplet interface for the higher viscosity ratios, which increases the tendency of the droplets to be pulled over each other.

Finally, the combined effects of geometrical confinement and viscosity ratio on droplet coalescence in shear flow are systematically mapped out and qualitatively explained. Furthermore, the generated dataset can serve as a reference for future development and validation of more accurate 3D numerical simulations of the coalescence of a droplet pair in bulk and confined conditions.

\section{ACKNOWLEDGMENTS}

R. Cardinaels is indebted to the Research Foundation Flanders (FWO) for a postdoctoral Fellowship. This work was partially funded by Onderzoeksfonds KU Leuven (GOA09/002).

\section{APPENDIX: FILM SHAPE}

Since the hydrodynamic force starts from zero at an orientation angle of $0^{\circ}$, reaches a maximum at an orientation angle of $45^{\circ}$, and decreases again to zero at an orientation angle of $90^{\circ}$ [see Eq. (7)], a transition from the spherical film regime to the flat film regime and back again can occur at certain orientation angles. Yang et al. (2001) argued that this transition will occur when the pressure inside the fluid film $\left(\sim F / a^{2}\right)$ becomes larger than the Laplace pressure $(\sim \Gamma / R)$. By filling in the hydrodynamic force [Eq. (7)] and the area of a parabolic/spherical film $\left(a^{2}=h R\right)$, Yang et al. (2001) found that, as a rough estimation, in bulk conditions the film will become flat when

$$
\sin \left(2\left(90^{\circ}-\theta\right)\right) \geq O\left(\frac{1}{f(\lambda) \operatorname{Ca}} \frac{h}{R}\right) .
$$

To use Eq. (A1) an estimation of the film thickness $h$ is needed. In order to calculate the range of angles for which the film is certainly flat, the maximum value of $h$ is used. An upper limit of the film thickness $h_{0}$ at the point where the transition from parabolic to flat film takes place can be estimated from simple geometric considerations, namely as the distance between the two surfaces of two truncated spheres at a distance $d=2 R$, where the radius of the film drainage area is estimated using Eqs. (7) and (8), resulting in the following formula [Yang et al. (2001)]:

$$
h_{0}=R-R \sqrt{1-f(\lambda) \cdot C a \cdot \sin \left(2\left(90^{\circ}-\theta\right)\right)} .
$$

As already explained, the upper limit of $h_{0}$ is needed. Since it is clear that the value of $h_{0}$ is maximum at $\theta=45^{\circ}$ this maximum value for $h_{0}$ is then used in Eq. (A1). This way it is 
estimated that the transition from a spherical to a flattened droplet shape occurs at a doublet orientation angle of $15^{\circ}$ (so almost right after apparent contact for most experiments in the present study), the transition back from a flattened to a spherical droplet shape is then estimated to occur at an angle of $75^{\circ}$, which corresponds to an offset of 0.966 . Since the value of $h$ keeps decreasing after apparent contact [see Eq. (9)] and since an upper limit is used for $h_{0}$, in reality the range of the flat film regime will even be larger. In confined conditions, the forces are larger and hence the tendency of the film to become flat will be larger. It must be noted that according to this simple scaling the range of angles leading to a flat film is not that sensitive to changes in the $C a$ number and the viscosity ratio. However, simulations performed by Yoon et al. (2007) showed that there are cases, depending on initial offset, viscosity ratio, and $\mathrm{Ca}$ number for which the film is always parabolic or parabolic for a considerable amount of time at high angles. Hence, it can be concluded that for a more accurate determination of the transition from a parabolic to a flat film, simulations of the full problem are necessary.

\section{References}

Aggarwal, N., and K. Sarkar, "Deformation and breakup of a viscoelastic drop in a Newtonian matrix under steady shear,” J. Fluid Mech. 584, 1-21 (2007).

Allan, R. S., and S. G. Mason, "Particle motions in sheared suspensions. XIV. Coalescence of liquid drops in electric and shear fields," J. Colloid Sci. 17, 383-408 (1962).

Bartok, W., and S. G. Mason, "Particle motions in sheard suspensions V. Rigid rods and collision doublets of spheres," J. Colloid Sci. 12, 243-262 (1957).

Batchelor, G. K., and J. T. Green, "The hydrodynamic interaction of two small freely-moving spheres in a linear flow field,” J. Fluid Mech. 56, 375-400 (1972).

Bayareh, M., and S. Mortazavi, "Binary collision of drops in simple shear flow at finite Reynolds numbers: Geometry and viscosity ratio effects," Adv. Eng. Software 42, 604-611 (2011).

Borrell, M., Y. Yoon, and L. G. Leal, "Experimental analysis of the coalescence process via head-on collisions in a time-dependent flow," Phys. Fluids 16, 3945-3954 (2004).

Bremond, N., A. Thiam, and J. Bibette, "Decompressing Emulsion Droplets Favors Coalescence," Phys. Rev. Lett. 100, 024501-1-024501-4 (2008).

Cardinaels, R., and P. Moldenaers, "Critical conditions and breakup of non-squashed microconfined droplets: Effects of fluid viscoelasticity," Microfluid. Nanofluid. 10, 1153-1163 (2011).

Caserta, S., and S. Guido, "Vorticity banding in biphasic polymer blends," Langmuir 28, 16254-16262 (2012).

Caserta, S., M. Simeone, and S. Guido, "A parameter investigation of shear-induced coalescence in semidilute PIB-PDMS polymer blends: Effects of shear rate, shear stress volume fraction, and viscosity," Rheol. Acta 45, 505-512 (2006).

Caserta, S., M. Simeone, and S. Guido, "Shear banding in biphasic liquid-liquid systems," Phys. Rev. Lett. 100, $1-4$ (2008).

Cassagnau, P., and F. Fenouillot, "Rheological study of mixing in molten polymers: 1-mixing of low viscous additives," Polymer 45, 8019-8030 (2004).

Chen, D., R. Cardinaels, and P. Moldenaers, "Effect of confinement on droplet coalescence in shear flow," Langmuir 25, 12885-12893 (2009).

Chesters, A. K., "The modelling of coalescence processes in fluid-liquid dispersions: A review of current understanding," Chem. Eng. Res. Des. 69, 259-270 (1991).

De Bruyn, P., R. Cardinaels, and P. Moldenaers, "The effect of geometrical confinement on coalescence efficiency of droplet pairs in shear flow,” J. Colloid Interface Sci. 409, 183-192 (2013).

Guido, S., and M. Simeone, "Binary collision of drops in simple shear flow by computer-assisted video optical microscopy,” J. Fluid Mech. 357, 1-20 (1998).

Guido, S., M. Simeone, and F. Greco, "Deformation of a Newtonian drop in a viscoelastic matrix under steady shear flow,” J. Non-Newtonian Fluid Mech. 114, 65-82 (2003). 
Guido, S., M. Simeone, and M. Villone, "Diffusion effects on the interfacial tension of immiscible polymer blends," Rheol. Acta 38, 287-296 (1999).

Hu, Y. T., D. J. Pine, and L. G. Leal, "Drop deformation, breakup, and coalescence with compatibilizer," Phys. Fluids 12, 484-489 (2000).

Jaeger, P. T., J. J. M. Janssen, F. Groeneweg, and W. G. M. Agterof, "Coalescence in emulsions containing inviscid drops with high interfacial mobility,” Colloids Surf., A 85, 255-264 (1994).

Jeffery, G. B., "The motion of ellipsoidal particles immersed in a viscous fluid," Proc. R. Soc. A Math. Phys. Eng. Sci. 102, 161-179 (1922).

Leal, L. G., "Flow induced coalescence of drops in a viscous fluid," Phys. Fluids 16, 1833-1851 (2004).

Loewenberg, M., and E. J. Hinch, "Collision of two deformable drops in shear flow," J. Fluid Mech. 338, 299-315 (1997).

Lyu, S.-P., F. S. Bates, and C. W. Macosko, "Coalescence in polymer blends during shearing," AIChE J. 46, 229-238 (2000).

Migler, K. B., "String formation in sheared polymer blends: Coalescence, breakup, and finite size effects," Phys. Rev. Lett. 86, 1023-1026 (2001).

Minale, M., P. Moldenaers, and J. Mewis, "Effect of shear history on the morphology of immiscible polymer blends," Macromolecules 30, 5470-5475 (1997).

Mousa, H., W. Agterof, and J. Mellema, "Determination of the orthokinetic coalescence efficiency of droplets in simple shear flow using mobile, partially mobile and immobile drainge models and trajectory analysis," Chem. Eng. Res. Des. 80, 345-354 (2002).

Park, C. C., F. Baldessari, and L. G. Leal, "Study of molecular weight effects on coalescence: Interface slip layer," J. Rheol. 47, 911-942 (2003).

Pathak, J. A., M. C. Davis, S. D. Hudson, and K. B. Migler, "Layered droplet microstructures in sheared emulsions: Finite-size effects,” J. Colloid Interface Sci. 255, 391-402 (2002).

Sarkar, K., and R. K. Singh, "Spatial ordering due to hydrodynamic interactions between a pair of colliding drops in a confined shear," Phys. Fluids 25, 051702 (2013).

Shardt, O., J. J. Derksen, and S. K. Mitra, "Simulations of droplet coalescence in simple shear flow," Langmuir 29, 6201-12 (2013).

Singh, R. K., and K. Sarkar, "Effects of viscosity ratio and three dimensional positioning on hydrodynamic interactions between two viscous drops in a shear flow at finite inertia," Phys. Fluids 21, 103303 (2009).

Son, Y., "Development of a novel microcompounder for polymer blends and nanocomposite," J. Appl. Polym. Sci. 112, 609-619 (2009).

Taylor, G. I., "The viscosity of a fluid containing small drops of another fluid," Proc. R. Soc. London A 138, 41-48 (1932).

Tretheway, D. C., M. Muraoka, and L. G. Leal, "Experimental trajectories of two drops in planar extensional flow," Phys. Fluids 11, 971-981 (1999).

Vananroye, A., P. Van Puyvelde, and P. Moldenaers, "Effect of confinement on droplet breakup in sheared emulsions," Langmuir 22, 3972-3974 (2006).

Verhulst, K., P. Moldenaers, and M. Minale, "Drop shape dynamics of a Newtonian drop in a non-Newtonian matrix during transient and steady shear flow,” J. Rheol. 51, 261-273 (2007).

Yang, H., C. C. Park, Y. T. Hu, and L. G. Leal, "The coalescence of two equal-sized drops in a two-dimensional linear flow," Phys. Fluids 13, 1087-1106 (2001).

Yoon, Y., F. Baldessari, H. D. Ceniceros, and L. G. Leal, "Coalescence of two equal-sized deformable drops in an axisymmetric flow," Phys. Fluids 19, 102102 (2007).

Yoon, Y., M. Borrell, C. C. Park, and L. G. Leal, "Viscosity ratio effects on the coalescence of two equal-sized drops in a two-dimensional linear flow," J. Fluid Mech. 525, 355-379 (2005).

Zinchenko, A. Z., "Calculation of hydrodynamic interaction between drops at low reynolds numbers," J. Appl. Math. Mech. 42, 1046-1051 (1978).

Zurita-Gotor, M., J. Bławzdziewicz, and E. Wajnryb, "Swapping trajectories: A new wall-induced cross-streamline particle migration mechanism in a dilute suspension of spheres," J. Fluid Mech. 592, 447-469 (2007). 